$\frac{46}{4} 6-9685(2)$

SANDIA REPORT

SAND96-1032 • UC-404

Unlimited Release

Printed May 1996

\title{
FWP Executive Summaries \\ Basic Energy Sciences \\ Materials Sciences Programs (SNL/NM)
}

\author{
G. A. Samara
}

Prepared by

Sandia National Laboratories

Albuquerque, New Mexico 87185 and Livermore, California 94550

for the United States Department of Energy

under Contract DE-AC04-94AL85000

Approved for public release, distribution is unlimited.
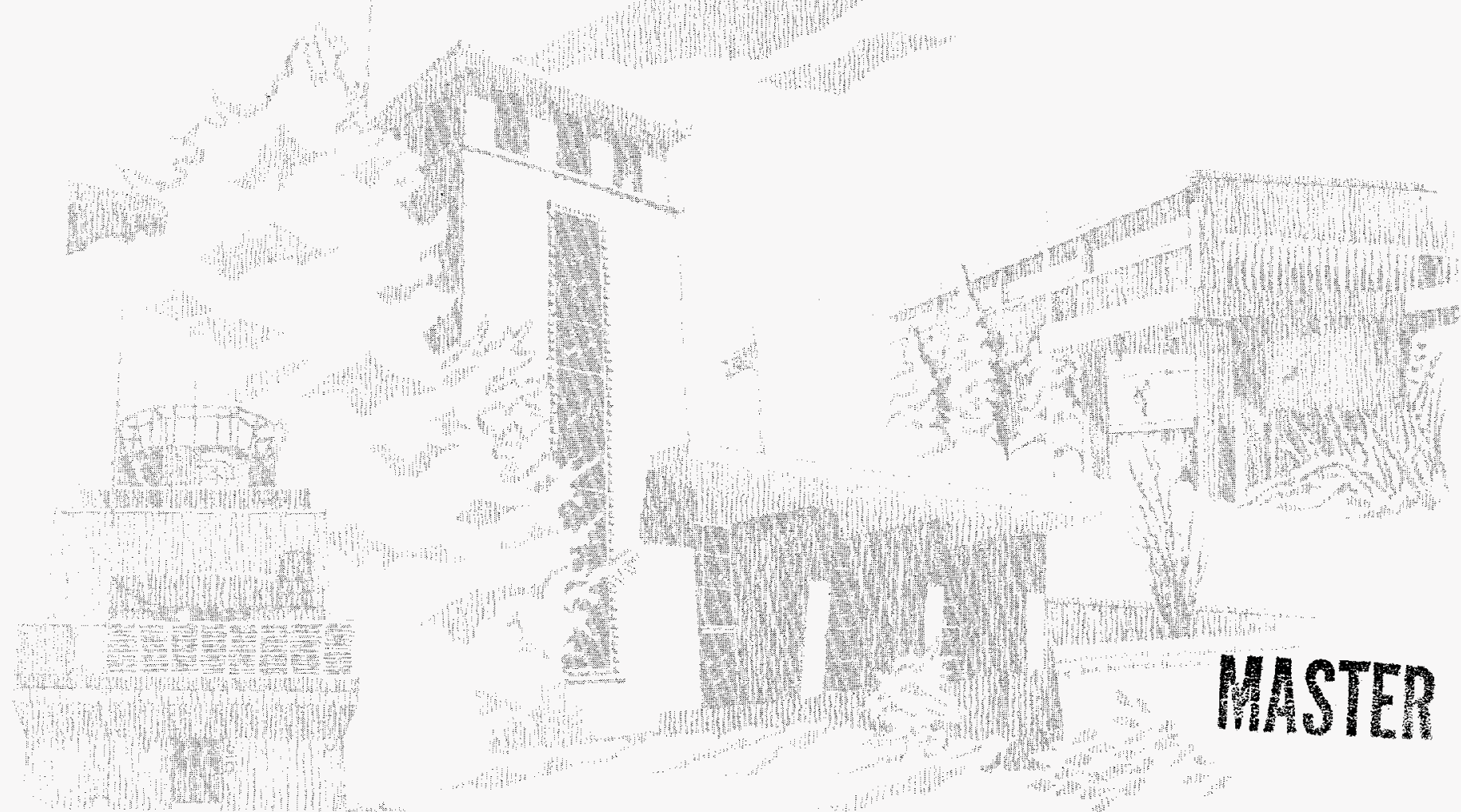
Issued by Sandia National Laboratories, operated for the United States Department of Energy by Sandia Corporation.

NOTICE: This report was prepared as an account of work sponsored by an agency of the United States Government. Neither the United States Government nor any agency thereof, nor any of their employees, nor any of their contractors, subcontractors, or their employees, makes any warranty, express or implied, or assumes any legal liability or responsibility for the accuracy, completeness, or usefulness of any information, apparatus, product, or process disclosed, or represents that its use would not infringe privately owned rights. Reference herein to any specific commercial product, process, or service by trade name, trademark, manufacturer, or otherwise, does not necessarily constitute or imply its endorsement, recommendation, or favoring by the United States Government, any agency thereof or any of their contractors or subcontractors. The views and opinions expressed herein do not necessarily state or reflect those of the United States Government, any agency thereof or any of their contractors.

Printed in the United States of America. This report has been reproduced directly from the best available copy.

Available to DOE and DOE contractors from

Office of Scientific and Technical Information

PO Box 62

Oak Ridge, TN 37831

Prices available from (615) 576-8401, FTS 626-8401

Available to the public from

National Technical Information Service

US Department of Commerce

5285 Port Royal Rd

Springfield, VA 22161

NTIS price codes

Printed copy: A04

Microfiche copy: A01 


\section{DISCLAMMER}

Portions of this document may be illegible in electronic image products. Images are produced from the best available original document. 
SAND96-1032

Unlimited Release

Printed May 1996

\title{
FWP EXECUTIVE SUMMARIES
}

\author{
BASIC ENERGY SCIENCES \\ MATERIALS SCIENCES PROGRAMS \\ (SNL/NM)
}

\author{
G. A. Samara \\ Program Manager
}

Sandia National Laboratories

Albuquerque, New Mexico

\begin{abstract}
This report provides an Executive Summary of the various elements of the Materials Sciences Program which is funded by the Division of Materials Sciences, Office of Basic Energy Sciences, U.S. Department of Energy at Sandia National Laboratories, New Mexico.
\end{abstract}

February 1996 


\section{Table of Contents}

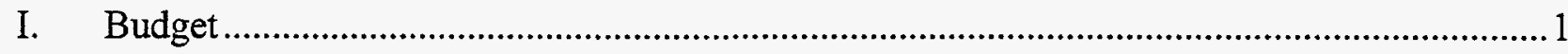

II. Capital Equipment Requests ............................................................................................

III. General Programmatic Overview and Institutional Issues...................................................

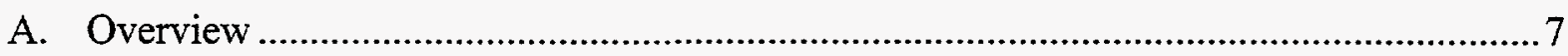

B. The DOE Center of Excellence for the Synthesis and Processing of Advanced Materials (CSP) ........................................................................................... 8

C. Interaction with DOE Technology Programs, Industry and Universities..........................9

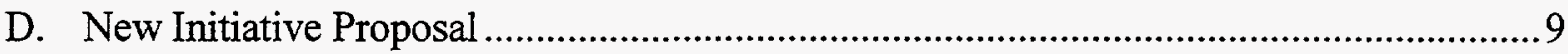

E. Program Reviews ................................................................................................

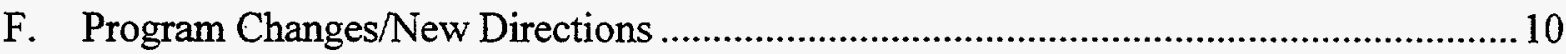

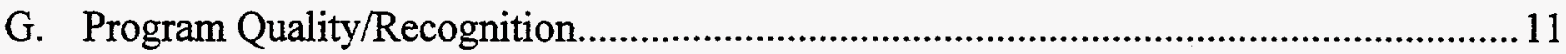

IV. The DOE Center of Excellence for the Synthesis and Processing

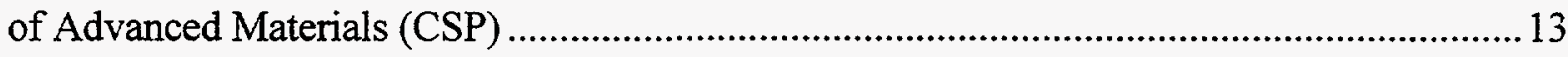

V. Interactions with DOE Technology Programs, Industry and Universities.............................21

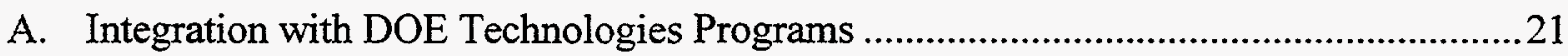

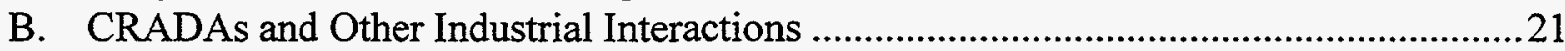

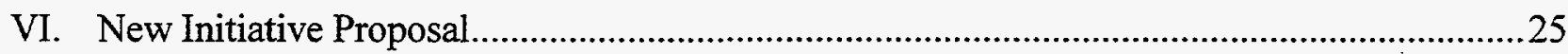

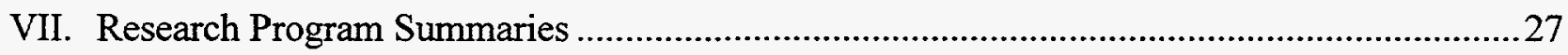

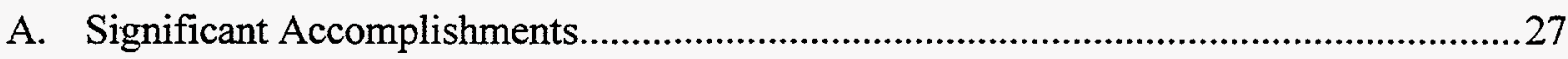

1. Structure and Dynamics of Inorganic Glasses ........................................................28

2. Capillary Phenomena in Porous Films...................................................................29

3. Direct Measurement and Atomistic Simulation of Molecular Mechanics................30

4. Quantification and Understanding of Extreme Strengthening in Multiphase Thin Film ....................................................................................

5. In Situ Measurement of CVD Growth Kinetics.....................................................32

6. Controlled, Nanoscale Defects for Enhanced Critical Current Density in $\mathrm{TlBaCaCuO}$ Thin Films via Furnace Annealing .....................................................33

7. First Principles Model for Crack Embrittlement by Liquid Metals ............................34

8. Time-Resolved Photoinduced Absorption in $\left(\mathrm{Al}_{\mathrm{x}} \mathrm{Ga}_{1-\mathrm{x}}\right)_{0.5} \mathrm{In}_{0.5} \mathrm{P}$ Fractal Quantum Well Heterostructures...........................................................................35

9. Direct Measurements of Si Surface Diffusion on Si(001) ........................................36

10. Pair Breaking in Boron Carbides' Small-Bipolaron Hopping …..............................37

11. Group V Inhibition of the GaAs and AlAs MOCVD Growth Rates .........................38

12. Semiconductor Nanoclusters as Potential Photocatalysts...........................................39 


\section{Table of Contents \\ (Continued)}

VII. Research Program Summaries (continued)... .27

B. Research Summaries

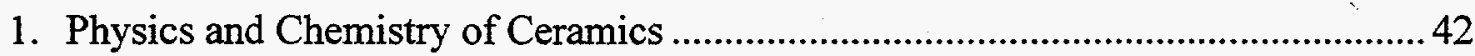

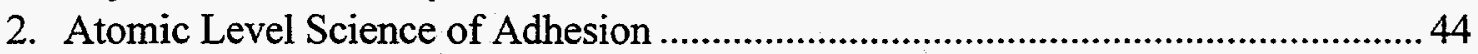

3. Wetting and Flow of Liquid Metals and Amorphous Ceramics

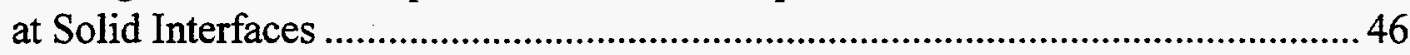

4. Energetic-Particle Synthesis and Science of Materials.............................................. 48

5. Advanced Growth Techniques and the Science of Epitaxy ....................................50

6. Artificially-Structured Semiconductor Materials Science.......................................52

7. Physics and Chemistry of Novel Superconductors.................................................5

8. Tailored Surfaces and Interfaces for Materials Applications ....................................56

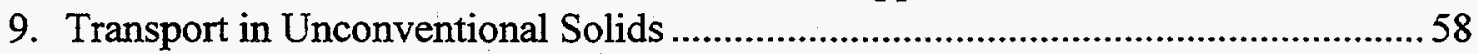

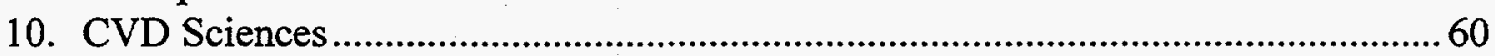

11. Synthesis and Processing of Nanoclusters for Energy Applications .......................62

12. Field-Structured Anisotropic Composites ................................................................64 


\section{Budget}

\section{Basic Energy Sciences/Materials Sciences Funding for FY95-98 Sandia National Laboratories - Albuquerque, New Mexico}

\begin{tabular}{|c|c|c|c|c|c|}
\hline $\begin{array}{c}\text { Active } \\
\text { Programs }\end{array}$ & Title & $\begin{array}{l}\text { AFP } \\
\text { FY95 } \\
\text { (SK) } \\
\end{array}$ & $\begin{array}{l}\text { AFP } \\
\text { FY96 } \\
\text { (\$K) } \\
\end{array}$ & $\begin{array}{l}\text { AFP } \\
\text { FY97 } \\
\text { (\$K) } \\
\end{array}$ & $\begin{array}{c}\text { Projected } \\
\text { FY98 } \\
\text { (\$K) } \\
\end{array}$ \\
\hline KC020101 & Physics \& Chemistry of Ceramics & 967 & 935 & 967 & 1035 \\
\hline $\mathrm{KC} 020102$ & Science of Adhesion & 481 & 447 & 481 & 515 \\
\hline KC020102 & Wetting and Flow of Liquid Metals & 96 & 257 & 400 & 428 \\
\hline $\mathrm{KC} 020103$ & Energetic Particle Synthesis & 775 & 753 & 775 & 829 \\
\hline $\mathrm{KC} 020103$ & Advanced Growth & 340 & 326 & 340 & 364 \\
\hline KC020105 & Artificially-Structured Semiconductors & 446 & 430 & 430 & 460 \\
\hline $\mathrm{KC} 020105$ & Field-Structured Anisotropic Composites & -- & 160 & 180 & 200 \\
\hline KC020105 & S\&P Center (Performance Meas.) & 97 & 88 & -- & -- \\
\hline KC020202 & Tailored Surfaces for Materials Application & 562 & 527 & 562 & 601 \\
\hline KC020202 & $\begin{array}{l}\text { Physics \& Chemistry of Novel } \\
\text { Superconductors }\end{array}$ & 539 & 505 & 539 & 577 \\
\hline $\mathrm{KC} 020205$ & Transport in Unconventional Solids & 490 & 475 & 490 & 524 \\
\hline KC020205 & High Temp. Superconductivity & 145 & 120 & 153 & -- \\
\hline KC020205 & Synthesis \& Processing Center & -- & 75 & 90 & 96 \\
\hline KC020303 & CVD Sciences & 651 & 612 & 651 & 697 \\
\hline \multirow[t]{2}{*}{$\mathrm{KC} 020303$} & Nanoclusters & 232 & 215 & 226 & 242 \\
\hline & TOTAL & 5,821 & 5,925 & 6,284 & 6,568 \\
\hline \multirow[t]{2}{*}{ Equipment } & - Programmatic & 1043 & 991 & 769 & 876 \\
\hline & - Awards & 200 & 50 & & \\
\hline
\end{tabular}

\section{New Initiative Proposal}

Covalently-Bonded Disordered Thin Film Materials $.500 \mathrm{~K}^{*}$

\footnotetext{
*This activity proposed for FY98 start up could start in FY97 if funds become available.
} 
This page intentionally left blank. 


\section{Capital Equipment Requests}

\section{Mid Year (FY96) Requests (In Priority Order)}

\section{High Temperature NMR Probe}

The electrical and mechanical properties of a glass ultimately depend on its chemical structure. The best strategy for a fundamental understanding of existing materials and intelligently designing new materials is to characterize the complex relationship between properties and molecular structure. Relaxation measurements provide a bridge between the macroscopic properties and microscopic structure of a glass. The diffusion of ionic species is related to the electrical properties of the glass, while the backbone motion of the chain is associated with mechanical properties such as viscosity and thermal expansion.

Both the diffusion of ionic species such as lithium, potassium and sodium, and the backbone motion of chains containing phosphorous or oxygen can be studied by NMR relaxation techniques. Currently we are limited to performing such measurements at temperatures of $140 \mathrm{C}$ or below. The new probe would enable us to investigate samples at temperatures up to $650 \mathrm{C}$. The increased temperature range would provide a more rigorous test of dynamic models as well as the ability to probe high temperature transitions which are currently inaccessible.

Although our initial studies will focus on BES programs, we expect that our increased understanding of processing, aging and reliability issues will impact DP and LDRD programs on glass/metal seals and on Smart Processing. This probe would also position us to investigate the high temperature behavior and degradation of organic materials.

Currently we are able to perform electrical measurements to $450 \mathrm{C}$ and viscosity measurements to $900 \mathrm{C}$. Our current limitation of $140 \mathrm{C}$ for NMR relaxation measurements means that we cannot compare macroscopic properties to microdynamical behavior over a broad temperature range. High temperatures enable us to go through important transitions in which macro/micro relationships become apparent.

\section{Scanning Probe Microscope for Characterization of Surface Structure}

An in situ atomic force/scanning tunneling microscope is needed for characterization of strained layer Group IV materials. The real space imaging information provided by this technique is a critical addition to the information determined by our surface diffraction techniques. The instrument will be used to study strain-induced surface roughening, ionassisted growth processes and cluster nucleation kinetics. 


\section{Major Equipment Needs for FY97 (In Priority Order)}

1. X-Ray Area Detector

$\$ 150 \mathrm{~K}$

For studies of diffuse x-ray scattering to probe atomic disorder and charge density waves, Local atomic displacements, perhaps related to large bipolarons, have been suggested as a key to high-temperature superconductivity. This area detector will permit quantitative studies of structural distortions in various cuprate superconductors and related materials.

2. Spectroscopic Ellipsometer

$\$ 200 K$

Ellipsometry is the gentle and expedient choice for the analysis of thin films made by sol-gel processing. By separately assessing physical and optical thickness, key insights have accrued in this program and elsewhere, inferred from refractive index models and dynamics. A crucial advantage of optical diagnostics is the potential to apply them in situ. Indeed, in previous years of this program, imaging ellipsometry was developed to assess sol-gel film deposition during dip coating. However, films designed for current and future applications often involve multilayer structures, such as multistage separation membranes and ferroelectric films. Conventional monochromatic, single-angle ellipsometry fails to resolve such internal structure, hence misses the physics and chemistry we seek. However, spectroscopic ellipsometers are now commercially available that have the capability to discern these subtleties.

Models of even simple sol-gel film drying suggest layered structure owing to "skinning" and similar phenomena. Only sporadic hints of such structure have been published. The new ellipsometer would help us to find and understand the conditions leading to films that vary through their thickness. Films with intentional multilayer structure or complex composition will be readily studied. Porous films partially saturated with liquids do not reveal where the liquid lies, an important input to ideas of stress development; however this information is available in principle through spectroscopic ellipsometry. Aerogel films exhibit enormous compliance as shown by this year's springback experiments, but it is not know whether the compactivity of the film is constant throughout; again spectroscopic ellipsometer promises answers.

Without the proposed equipment, variations through thickness must be observed through tedious microscopy and microprobe analysis. The cost of sample section preparation alone ( $\$ 1000$ per sample) precludes freely exploratory chemistry or processing. Moreover, situations requiring in situ analysis, such as during capillary stressing, are virtually impossible to address by microscopy (at least we have not found a way).

3. Work Station for Molecular Modeling of Materials Synthesis and Structure

$\$ 60 \mathrm{~K}$

In the fabrication of multicomponent ceramics by chemical methods, the properties of the ceramics are determined by the characteristics of the precursors, and how the precursors evolve into the desired ceramic phase. Therefore, to effectively prepare materials that possess properties which are optimized for a given application, it is important to be able to 
understand the synthetic routes by which particular precursors may be obtained, and to have a fundamental knowledge of the mechanisms by which precursor structure impacts final material properties.

Historically, optimization of material structure and properties has required extensive experimental investigation to develop suitable processing routes and an understanding of processing - property relationships. This has severely hindered the "design" of effective synthetic routes and the development of new, high technology materials. Recently, however, computer simulation software that would allow for the molecular modeling of materials synthesis has become commercially available.

However, while such software is available, very little has been done to apply its use to the processing of complex ceramics by chemical methods. After developing our own interatomic potentials, we would utilize the available software, in particular, the molecular dynamics simulation packages, to design new aqueous co-precipitation and sol-gel chemprep routes for multicomponent ceramic production. The focus of the modeling would be on understanding formation mechanisms for metalorganic and oxide precursors, and predicting precursor structural evolution by modeling decomposition pathways and phase transformation behavior. We plan to carry out verification of the synthesis models by experimentation with the newly devised routes. Targeted material products would include oxide powders and thin films for applications ranging from next generation current stacks to separation membranes.

This program would take Sandia to a "higher level" in our mission of materials synthesis based on scientific understanding. If successful, the models generated would provide a powerful tool for developing synthesis schemes for producing materials by design that have properties optimized for different applications. 
This page intentionally left blank. 


\section{General Programmatic Overview and Institutional Issues}

\section{A. Overview}

The goals of the BES Materials Sciences Program at Sandia National Laboratories/New Mexico (SNL/NM) are:

- Perform basic, forefront interdisciplinary research that also demonstrates relevance of basic science to technology.

- Choose programs which support DOE interests and are complementary to Sandia's multiprogram laboratory mission.

- Perform research in a setting which enhances technological impact because of Sandia's spectrum of coordinated basic research, applied research and development engineering.

- Take advantage of a wide range of large, capital-intensive research facilities not usually found at universities.

Our BES Materials Sciences Program has the central theme of Scientifically Tailored Materials. The major objective of this program is to combine Sandia's expertise and capabilities in the areas of solid state sciences, advanced atomic-level diagnostics and materials synthesis and processing science to produce new classes of tailored materials as well as to enhance the properties of existing materials for U.S. energy applications and for critical defense needs.

Current research in this program at SNL/NM includes the physics and chemistry of ceramics synthesis and processing, the use of energetic particles for the synthesis and study of materials, high-temperature and organic superconductors, tailored surfaces and interfaces for materials applications, chemical vapor deposition sciences, artificially-structured semiconductor materials science, advanced growth techniques for improved semiconductor structures, transport in unconventional solids, atomic-level science of interfacial adhesion, and the synthesis and processing of nano-size clusters for energy applications. Considerable synergism exists among these program elements, and most of them share a number of common themes based on either the science or the use of common synthesis and processing approaches. These themes are: Synthesis and Processing, Epitaxial Growth, Surfaces and Interfaces, and the use of Energetic Beams. Several of the technical activities of the program have been incorporated into the DOE Center of Excellence for the Synthesis and Processing of Advanced Materials.

Two new research activities initiated in late FY 94 after DMS allocated some funding for each are progressing well. The first is a collaboration with the National Renewable Energy Laboratory on "Composition Modulated Semiconductor Structures for Photovoltaic and Optical Technologies." DMS provided post doctoral support for this work in FY94 and FY95 and recently increased the funding to $\$ 200 \mathrm{~K}$ for each lab for FY96. The work deals with compositional structuring and alloy ordering in III-V semiconductors to enhance their performance for 
photovoltaic and other optical applications. Programmatically this activity is included as one of the tasks under our core program on Artificially Structured Semiconductor Materials Science.

The second activity is on "Wetting and Flow of Liquid Metals and Amorphous Ceramics at Solid Interfaces." The goal of this work is to perform experiments and model the atomic-scale processes that control fluid flow at solid interfaces and use these understandings to develop predictive models for macroscopic wetting and flow behavior. The results of this work are expected to be relevant to the Materials Joining Project of the Synthesis and Processing Center, and interactions with the Project will be initiated. Part of our emphasis in FY95 was to focus on developing collaborative interactions with our university partner (Uzi Landman - Georgia Tech). We transferred $20 \%$ of the $\$ 96 \mathrm{~K}$ provided in FY95 to Georgia Tech to conduct molecular dynamics simulations. This cooperative work has allowed us to directly compare measured viscoelastic properties of monolayer films with simulations for the identical system. We are also using a Sandia-funded LDRD project on thin-film viscoelasticity measurement techniques to leverage our BES funds and get this new project off to a quick start.

DMS has also recently provided start-up funds ( $\$ 160 \mathrm{~K}$ for FY96) to initiate research on "FieldStructured Anisotropic Composites." This work builds on and extends the ideas presented to DMS in our FY95 New Initiatives proposal. In this work we propose to use external electric and magnetic fields to create novel ceramic/polymer and metal/polymer nanocomposites with one- and two-dimensional anisotropy, by polymerizing a continuous network-forming phase around particles having an electric permittivity or magnetic permeability mismatch, while subjected to an applied electric or magnetic field. The applied field will polarize the particles, inducing strong anisotropic forces that have components parallel and perpendicular to their line of centers. The resultant fieldstructured nanocomposites will have highly anisotropic mechanical and transport properties, and can be made of a wide variety of materials. They have numerous potential applications in energy and defense programs.

Our interdisciplinary program utilizes a broad array of sophisticated, state-of-the-art experimental capabilities provided by other programs as well as this program. The major leveraged capabilities include several molecular beam epitaxy and chemical vapor deposition facilities, a broad range of materials synthesis and processing facilities, clean rooms, ion-beam accelerators, laser-based diagnostics, advanced optical and surface spectroscopies, unique combined high-pressure/lowtemperature/high-magnetic-field facilities, and electron, scanning tunneling and atomic force microscopies.

\section{B. The DOE Center of Excellence For The Synthesis and Processing of Advanced Materials (CSP)}

In addition to being involved in the technical program of CSP, Sandia is responsible for the overall coordination of Center activities. Summaries of our coordination efforts and a description of the projects which constitute the current technical focus of the Center are given in Section IV, p. 13. 


\section{Interaction with DOE Technology Programs, Industry and Universities}

Our DMS-funded program is strongly coupled to DOE technologies programs at Sandia and has been responsible fully or in part for the generation of a large number of cooperative research and development agreements (CRADAs) with industrial partners. We also have several less formal collaborations with industry and broad collaborations with universities. A summary is provided in Section V, p. 21.

\section{New Initiative Proposal}

This year we have submitted a new proposal entitled "Covalently-Bonded Disordered Thin Film Materials." The goal of this proposal is to closely couple a multi-disciplinary materials research program with state-of-the-art advances in software and hardware at Sandia to develop a fundamental understanding of covalently-bonded disordered thin film materials. These materials are amorphousto-nanocrystalline structures made from light atomic weight elements (B, C, and N). We have initiated our work through applications-focused laboratory directed R\&D (LDRD) and industrial CRADA programs. These efforts, while successful, have demonstrated the overwhelming need for an in-depth conceptual understanding of the relationship between growth energetics, atomic structures and materials properties. We will use first-principles theory, density-functional electronic structure geometry optimizations, to determine representative atomic configurations. This will allow direct comparison with experimental measurements, such as vibrational and optical spectroscopies, and radial distribution functions. The ability to relate theory with experiment serves both to validate the theory and to provide physical interpretations to the spectroscopies. A further goal is to gain an understanding of the relationship between the growth energetics and the resulting thin film structures, resulting in the ability to design engineered materials for specific applications.

\section{E. Program Reviews}

\section{External Review Panel}

Sandia's Materials Science and Technology (MS\&T) Council sponsors an annual external review of our MS\&T programs. This review looks into the motivation, organization, management and technical quality of the program. Our DMS program was part of the 1995 review that took place on Feb. 13-14, 1995. The high quality of this program and its integration with other technologies programs were noted by the Review Panel. The Panel also recommended stronger interactions with energy programs at Sandia. Panel members were: John Bravman (Stanford); Jeff Bullington (AMMPEC); David Crawford (United Technologies); Paul Fleury (AT\&T Bell Labs); Ross Lemons (Los Alamos); James Nottke (DuPont Central Research Labs); and Kathleen Taylor (GM Research). 


\section{DMS Management On-Site Visits}

- On April 14, 1995 Dr. Arthur Dennison of DMS visited and discussed Sandia programs supported by the Metallurgy and Ceramics branch.

- On May 3, 1995 Dr. Jerry Smith of DMS visited and discussed Sandia programs supported by the Solid State Physics and Materials Chemistry branch.

\section{Germantown Management Review}

The annual management review of our program was held in Germantown on March 8, 1995.

\section{In-House Management Reviews}

Each element of our program is evaluated on a continuing basis with respect to objectives and performance by the responsible line management. Each involved participant is evaluated on his/her contributions in a formal annual review process with feedback.

\section{F. Program Changes/New Directions}

The Ceramics Program continued to develop and sharpen its new focus on understanding the chemical and physical processes that link precursors to the properties of ceramics and glasses. The emphasis is on three main tasks: Porous Materials, Complex Precursors and Glass Dynamics. A small effort on statistical treatments of powder compaction was also begun last year. All tasks combine coupled experimental and theoretical efforts.

The title of the "Boron-Rich Solids" program was changed to "Transport in Unconventional Solids." With this change we would continue to study boron-rich solids. However, we would gain a stronger charter to apply insights gained from studying borides to understanding transport in other unconventional solids. Examples include the clathrates based on networks of $\mathrm{Si}$ and $\mathrm{Ge}$ atoms and the pyrochlores. Like boron carbides, heavily doped clathrates are reported to have Seebeck coefficients that are orders of magnitude larger than would be expected from their large carrier densities. They are potentially very attractive for thermoelectric applications.

Sandia's newly developed Atom Tracker greatly expands our ability to observe atom and vacancy motion on surfaces. By "locking-on" to the position of a selected atom (or vacancy), this new version of a scanning tunneling microscope can follow each atomic step of a complex diffusion path. In this way the diffusing atom itself becomes a probe of surface structure and chemistry. The product of a Sandia LDRD project, this technique provides new opportunities to examine atomscale dynamics that could strongly complement ongoing Field Ion Microscopy and theoretical simulations in the Tailored Surfaces and Interfaces for Materials Applications project. With the arrival of the Low Energy Electron Microscope (purchased with FY95 BES Capital Funds) this project will be at the forefront of experimental and theoretical approaches for modeling atom-scale surface and interface processes. 


\section{G. Program Quality/Recognition}

1995 was another excellent year in terms of the significant accomplishments and productivity of our DMS program (as detailed in Section VII of this Executive Summaries booklet) and in terms of the professional recognition received by our staff, both of which reflect on the quality of our BES program.

Jeff Brinker was the recipient of the American Chemical Society's 1996 Ralph K. Iler Award in Colloid Chemistry of Materials, sponsored by DuPont. The award recognizes contributions toward a fundamental understanding of sol-gel processing, especially thin film deposition, and the synthesis and processing of novel porous materials: from ambient pressure aerogels to amorphous molecular sieves.

Brinker, Hurd, Schunk, Frye, Samuel, and Ashley, along with UCLA collaborators, were named winners of the 1995 BES award for Sustained Outstanding Research in Metallurgy and Ceramics for their work on "Fundamentals of Sol-Gel Thin Film Deposition". Their work over several years on the fundamentals of sol-gel film formation has revealed a number of surprises, including the importance of capillary stress phenomena, evaporation singularities, thin aerogel films, and surface acoustic wave absorption isotherms of films. These phenomena have been exploited to prepare diverse thin film structures ranging from aerogels processed under ambient conditions to amorphous molecular sieves that discriminate between molecules less than $0.02 \mathrm{~nm}$ different in size.

We also organized conferences, symposia, chaired sessions, wrote review papers, and presented numerous invited papers at national and international conferences (see individual program summaries in Section VII-B for details). 
This page intentionally left blank.

$-12-$ 


\section{The DOE Center of Excellence for the Synthesis and Processing of Advanced Materials (CSP)}

\section{Overview}

The DOE Center of Excellence for the Synthesis and Processing of Advanced Materials is a distributed center for promoting coordinated, cooperative research partnerships related to the synthesis and processing of advanced materials. It was established by DOE's Division of Materials Sciences, Office of Basic Energy Sciences and the DOE Laboratories in recognition of the enabling role of materials synthesis and processing to numerous fabrication- and manufacturing-intensive technologies. The participants include investigators from $12 \mathrm{DOE}$ national laboratories, universities and the private sector. The Center has a technological perspective which is provided by a Technology Steering Group. A set of performance measures for the Center is being developed.

The current emphasis of the Center is on seven focused multilaboratory projects which draw on the complementary strengths of the member institutions in their ongoing research programs. These nine projects were selected on the basis of the following criteria: (1) scientific excellence, (2) clear relationship to energy technologies, (3) involvement of several laboratories, (4) strong existing or potential partnerships with DOE Technologies-funded programs, and (5) strong existing or potential partnerships with industry.

The seven projects are: (1) Metals Forming, (2) Materials Joining, (3) Microstructural Engineering with Polymers, (4) Tailored Microstructures in Hard Magnets, (5) Processing for Surface Hardness, (6) Mechanically Reliable Surface Oxides for High Temperature Corrosion Resistance, and (7) High Efficiency Photovoltaics.

The member laboratories of the Center are: Ames Laboratory (Ames), Argonne National Laboratory (ANL), Brookhaven National Laboratory (BNL), Idaho National Engineering Laboratory (INEL), University of Illinois Frederick Seitz Materials Research Laboratory (UI/MRL), Lawrence Berkeley National Laboratory (LBNL), Lawrence Livermore National Laboratory (LLNL), Los Alamos National Laboratory (LANL), National Renewable Energy Laboratory (NREL), Oak Ridge National Laboratory (ORNL), Pacific Northwest National Laboratory (PNNL), and Sandia National Laboratories (SNL). The Center also includes appropriate university grant research.

\section{Objective}

The overall objective of the Center is,

"To enhance the science and engineering of materials synthesis and processing in order to meet the programmatic needs of the Department of Energy and to facilitate the technological exploitation of materials". 
Synthesis and processing are those essential elements of materials science and engineering (MS\&E) that deal with (1) the assembly of atoms or molecules to form materials, (2) the manipulation and control of the structure at all levels from the atomic to the macroscopic scale, and (3) the development of processes to produce materials for specific applications. Clearly, S\&P represent a large area of MS\&E that spans the range from fundamental research to technology. The goal of basic research in this area ranges from the creation of new materials and the improvement of the properties of known materials, to the understanding of such phenomena as diffusion, crystal growth, sintering, phase transitions, etc., in relation to S\&P. On the applied side, the goal of S\&P is to translate scientific results into useful materials by developing processes capable of producing high quality, cost-effective products.

The Center's emphasis is on the elucidation and application of fundamental S\&P principles directed toward the rapid improvement or development and ultimate utilization of advanced materials. In order to meet its overall objective, the Center has the following specific objectives:

1) Develop synthesis and processing methodologies to control structure, and thereby materials properties, from the atomic to the macroscopic scale.

2) Discover and develop high-payoff, advanced materials.

3) Integrate fundamental scientific principles with the concurrent development of synthesis and processing in collaboration with DOE technologies-funded programs and with industry.

\section{The Center's Technology Steering Group}

A Technology Steering Group (TSG) for the Center has been established. The role of TSG is to become familiar with the Center's technical activities and comment on their technological value, provide information from an industrial perspective, help identify technological barriers, influence the direction of the Center's programs, and help develop ideas which can make the Center more effective.

Current TSG membership is as follows:

Member
Dr. David J. Beecy
Dr. Thomas C. Clarke
Dr. David W. Johnson, Jr.
Dr. Hylan B. Lyon
Dr. Neil E. Paton
Dr. John Stringer

\section{Center Performance Measures}

A small project has been set up to develop performance measures for the Center. The project, under the direction of Dr. Gretchen Jordan of Sandia National Laboratories, is researching methods for annual performance measurement that capture progress and socio-economic impacts in addition to measures of the quality of science. A model will be documented for possible transfer to other BES activities. 


\section{Materials and Processes Focus of the Center}

The current emphasis of the Center is on seven focused multilaboratory projects. Each of the projects is coordinated by an appropriate representative from one of the participating institutions. The overall Center coordinator is:

George A. Samara:

Phone:

Fax:
( $S N L / N M)$

(505) 844-6653

(505) $844-4045$

A brief description of each project follows:

\section{Metals Forming}

Participating Labs:

Coordinator:

Phone:

Fax:
Ames, LBNL, LLNL, LANL, UI/MRL, ORNL, PNNL, SNL/CA, SNL/NM Michael E. Kassner (LLNL)

(541) $737-7023$

(541) $737-2600$

This project is motivated by the goal of improving fuel efficiency in transportation systems. Achieving this goal requires the use of light weight structural materials which in turn necessitates consideration of aluminum alloys. Unfortunately, compared to steels, $\mathrm{Al}$ alloys are more difficult to form and exhibit rougher surfaces after forming.

The major objective of the project is to concentrate on issues which determine deformation mechanisms and control the formability of light-weight alloys. Current emphasis is on dynamic recrystallization in $\mathrm{Al}-\mathrm{Mg}$ alloys with three complementary tasks: (1) the development of heterogeneous dislocation substructures and high-angle interfaces, (2) particle-stimulated recrystallization and in-situ studies, and (3) the development of constitutive equations to predict dynamic recrystallization and hot rolling.

\section{Materials Joining}

Participating Labs: Coordinator: Ames, INEL, LBL, LLNL, ORNL, PNNL, SNL/CA, SNL/NM Phone: R. B. Thompson (Ames)

Fax: (515) 294-9649

(515) $294-4456$

Materials joining is an enabling technology in virtually all industrial sectors, and often the reliability of joints is the factor that limits performance. Welding is an old technology, but weld failures are common and some technologically important materials such as aluminum alloys are difficult to weld. Advanced high temperature ceramics have tremendous potential in energy and related 
technologies, but there are no reliable methods of joining them. These realities provide the motivation for this project.

The project consists of two tasks. The first entitled "The effects of Gradients on Weld Reliability and Performance," uses advances in experimental, analytical and computational tools to develop an integrated and quantitative understanding of the origin and extent of gradients in composition, stress, microstructure and properties which occur during various welding processes. Strategies will also be developed to control these gradients which are often the cause of failure. Initial emphasis is on $\mathrm{Al}-\mathrm{Cu}$ alloys and on $\mathrm{Fe}-\mathrm{Ni}-\mathrm{Cr}$ alloys.

The second task, "Ceramics and Dissimilar Materials Joining," focuses on critical issues in the non-welding joining area which include property mismatch between members to be joined; use temperature limitation; poor wetting, adhesion and/or chemical bonding; and manufacturing and/or joint reliability. Some of the initial emphasis is on silicon carbide joining, an area of strong interest to the Fossil Energy program. This part of the work is being done in collaboration with research sponsored by the Office of Advanced Research, Fossil Energy program.

\section{Tailored Mcrostructures in Hard Magnets}

Participating Labs: Ames, ANL, BNL, INEL, LBL, LLNL, LANL, ORNL

Coordinator: $\quad B o b$ Dunlap (ANL)

Phone: (708) 252-4925

Fax: $\quad$ (708) $252-4798$

Improvements in the properties of permanent (or hard) magnetic material can lead to lighter, more efficient and longer life motors for energy, transportation and many other industries. A figure of merit for permanent magnet materials is the maximum energy product, $W$. In some of the best current commercial materials $\mathrm{W}$ is $\leq 50 \%$ of its theoretical value. The problem is generally attributed to a lack of understanding of the role of microstructure in determining magnetic properties. Other limitations of current commercial magnetic materials are relatively poor mechanical and corrosion-resistant properties. These properties are also determined largely by microstructure.

The overall objective of this project is to improve hard magnets by understanding, in terms of the microstructures achieved, the magnetic and mechanical properties of materials produced by a number of synthesis and processing (S\&P) approaches.

Initial focus is on the technologically important material $\mathrm{Nd}_{2} \mathrm{Fe}_{14} \mathrm{~B}$ as a model system. Specifically, this material is being produced in single crystal, powder, bulk and thin film forms and characterized by state-of-the-art tools. The microstructures developed by the different S\&P methods are being compared and modeled. The relationship between microstructure and domain wall pinning, magnetic properties and mechanical properties is being determined. The ultimate goal is to identify $\mathbf{S} \& \mathrm{P}$ approaches which optimize material properties for specific applications. 
Participating Labs: $\quad$ Ames, BNL, INEL, UI/MRL, LBL, LLNL, PNL, SNL/NM

Coordinator:

Phone:

Gregory J. Exarhos (PNL)

Fax:

(509) $375-2440$

(509) $375-2186$

The manipulation of polymer chain structure underlies the current, "age of polymers." Although discoveries of new polymers continue, it is widely recognized that emerging technologies will use multiphase polymers, structurally designed to achieve properties inaccessible by simple, singlecomponent materials. These materials will exploit phase separation phenomena, restricted to submicron scales by clever design of precursors and processing protocol. This project seeks to establish the scientific basis for tailoring such structurally engineered materials.

The goal of this project is to develop new multiphase materials that retain the processability of organic polymers but share the properties normally associated with inorganic materials. The project seeks a foundation for multiphase polymers similar to the knowledge base in small-molecule chemistry that underlies the current generation of commodity polymers. Initial emphasis is on the development of advanced synthesis and processing approaches for mesostructured polymers, polymer blends and polymer ceramic/glass composites.

\section{Processing for Surface Hardiness}

Participating Labs: $\quad L B L, L L N L, L A N L, O R N L, S N L / C A, S N L / N M$

\section{Coordinator:}

Phone:

Fax:

\section{J. B. Roberto (ORNL)}

(423) $576-0227$

(423) $574-4143$

There exists a broad range of applications for which the ability to produce an adherent, hard, thin, wear-resistant coating plays a vital role. These applications include engine and machine components, orthopedic devices, textile manufacturing components, hard disk media, micromachined sensors and actuators, optical coating, and cutting and machining tools (e.g., punches, taps, scoring dies, and extrusion dies). Emphasis is being placed on development and improvement of processes which are environmentally benign and which provide flexible control over the surface structure and chemistry.

Plasma-based processing is an important component of processes used for the applications listed above. The ability to provide flux, energy, and temporal control of a variety of ions, which is characteristic of plasma-based processing, provides the means to tailor surface hardness and other tribological properties.

The goal of the project is to address critical issues which limit the use of plasma-based processing for surface hardness. Initial emphasis is on plasma ion immersion processing (PIIP), a relatively inexpensive non-line-of-sight-implantation process capable of treating complex-shaped targets without complex fixturing, and on boron-based superhard coatings where the focus is on cubic boron nitride and boron suboxides. 
Participating Labs: $\quad A N L, I N E L, L B L, L L N L, O R N L$

Coordinator: $\quad$ Linda L. Horton (ORNL)

Phone: $\quad$ (423) $574-5081$

Fax: $\quad$ (423) $574-4066$

Protection from corrosion and environmental effects arising from deleterious reactions with gases and condensed products is required to fully exploit the potential of advanced high-temperature materials designed to improve energy efficiency and minimize deleterious environmental impact. The resistance to such reactions is best afforded by the formation of stable surface oxides that are slow growing, sound, and adherent to the substrate and/or by the deposition of coatings that contain or develop oxides with similar characteristics. However, the ability of brittle ceramic films and coatings to protect the material on which they are formed or deposited has long been problematical, particularly for applications involving numerous or severe high temperature thermal cycles or very aggressive environments. This lack of mechanical reliability severely limits the performance or durability of alloys and ceramics in many high-temperature industrial applications and places severe restrictions on deployment of such materials. The beneficial effects of certain alloying additions on the growth and adherence of protective oxide scales on metallic substrates are well known, but satisfactory broad understandings of the mechanisms by which scale properties and coating integrity (that is, corrosion resistance) are improved by compositional, microstructural, and processing modifications are lacking.

The objective of this task is to systematically generate the knowledge required to establish a scientific basis for the design and synthesis of improved (slow growing, adherent, sound) protective oxide coatings and scales on high temperature materials without compromising the requisite bulk material properties. Specific objectives are to (1) systematically investigate the relationships among substrate composition and properties and scale/coating failure, and (2) identify conditions leading to more damage-tolerant coatings and scales that are amenable to legitimate synthesis routes. The initial emphasis is on alumina scales and coatings, and the work is co-sponsored by the Office of Advanced Research, Fossil Energy Program. Some of the work in the project is also in collaboration with the Electric Power Research Institute (EPRI).

\section{High Efficiency Photovoltaics}

Participating Labs: Ames, ANL, BNL, INEL, UI/MRL, LBL, LLNL, NREL, ORNL, PNL, SNL/CA, SNL/NM, Caltech, MIT, SUNY, U.Florida, UCSB, U.Utah, Washington State $U$.

Coordinator: $\quad$ S. K. Deb/J. Benner (NREL)

Phone: $\quad$ (303) 384-6405/(303) 384-6496

Fax: $\quad$ (303) $384-6481$

Advances in the technology of solar photovoltaic (PV) energy conversion are critically dependent on the fundamental understanding of the synthesis and properties of the materials that compose solar cells. Reduced cost, improved conversion efficiency, and long-term stability are the major 
objectives of the DOE Photovoltaics Program. Thin-film semiconductor materials and device technologies are key to achieving these objectives. Currently, there are several important classes of thin-film PV materials at various stages of research and development, but in all cases there is a lack of understanding of the fundamental scientific issues associated with each of these technologies. Therefore, this program is motivated by the scientific exploration of new solid-state physics as it relates to photon absorption and carrier transport, novel materials synthesis techniques, the characterization and control of defect structures, and ultimately designs of new material architectures.

The project is focused on two areas: (1) Silicon-Based Thin Films, in which key scientific and technological problems involving amorphous and polycrystalline silicon thin films will be addressed; and (2) the Next Generation Thin Film Photovoltaics, which will be concerned with the possibilities of new advances and breakthroughs in the materials and physics of photovoltaics using non-silicon-based materials. 
This page intentionally left blank.

$-20-$ 


\section{Interaction with DoE Technology Programs, Industry and Universities}

\section{A. Integration with DOE Technologies Programs}

One of the primary goals of our DMS program at Sandia is to choose research areas which support DOE technology interests and are complementary to the Laboratories' multi-program mission. Consequently, all DMS-supported research is strongly integrated with our other R\&D activities in support of defense, energy and environmental programs. Specific areas where DMS research impacts DOE technologies needs include: materials synthesis and processing, long-term materials stability and reliability of components, reduction of friction and wear, control of impurities and defects in microelectronics and photovoltaics, radiation hardness of microelectronics and photonics, improved materials joining, advanced diagnostics and sensors, advanced separations membranes, novel catalysts and environmental remediation concepts. The accompanying table summarizes DOE technologies interests and interactions with our DMS program.

\section{B. CRADAs and Other Industrial Interactions}

The research results and capabilities of our DMS-sponsored program have been responsible fully or in part for the generation of over 30 cooperative research agreements or contracts with industrial partners. Our DMS-sponsored research has also resulted in numerous other less formal, but significant industrial interactions and/or technology transfer activities. Details were given in last year's Executive Summaries. 


\section{Sandia National Laboratories/New Mexico BES/MS Program \\ Complementary DOE Technologies Support/Interest}

\section{BES/MS Project}

1. Physics and Chemistry of Ceramics

Assink et al

2. Atomic Level Science of Adhesion

Michalske et al

3. Wetting and Flow of Liquid Metals and Amorphous Ceramics at Solid Interfaces Michalske et al

4. Energetic-Particle Synthesis and Science of Materials Barbour et al

5. Advanced Growth Techniques and the Science of Epitaxy Chason et al

6. Artificially-Structured Semiconductor Materials Science

Gourley et al

7. Physics and Chemistry of Novel Superconductors Aselage et al

\section{Related/Interested}

DOE Technologies

FE/METC

DP

DP

FE

DP

DP

MFE

DP

EE

DP

EE/STEPS

DP

\section{Relationship}

Separations membranes (complementary funding)

Aerogels, powder composition, ceramic processing composites (complementary funding)

Bonding strength and reliability (complementary funding)

Ceramic joining

Metal and Ceramic joining (complementary funding)

Hard surface layers; plasma processing (complementary funding)

First wall materials and diagnostics

MBE growth and in-situ monitoring (complementary funding)

Photovoltaic materials

Photonic materials for devices

(complementary funding)

Phase equilibria and processing of $\mathrm{Tl}$ high

Tc superconductors

Materials for high Tc devices

(complementary funding) 


\section{Sandia National Laboratories/New Mexico BES/MS Program \\ Complementary DOE Technologies Support/Interest \\ (Continued)}

\section{BES/MS Project}

8. Tailored Surfaces and

Interfaces for Materials

Applications

Kellogg et al

9. Boron-Rich Solids

Aselage et al

10. CVD Sciences

Esherick et al

11. Synthesis and Processing of Nanoclusters for Energy

Applications

Wilcoxon et al

12. Field-Structured Anisotropic

Composites

Martin et al
Related/Interested

DOE Technologies

DP

MFE

$\mathrm{EE}$

DP

DP

EE

FE/PETC

DP

EM

EE

DP

\section{Relationship}

Surface/interface stability; aging, reliability (complementary funding)

First wall materials

High temperature thermoelectrics

Neutron detectors; thermoelectrics

(complementary funding)

Materials growth; reactor design; in-situ diagnostics (complementary funding)

Photovoltaic materials

Coal catalysis

Novel device concepts (complementary funding)

Waste recovery

Vibration damping, controls, clutches

Controls, vibration damping, component encapsulants 
This page intentionally left blank. 


\section{New Initiative Proposal}

\section{Covalently-Bonded Disordered Thin Film Materials}

\section{Principal Investigators and Collaborators}

P.I.'s: SNL/NM M. P. Siegal, E. B. Stechel, T. A. Friedmann, P. A. Schultz, D. R. Tallant Collaborators: SNL/CA T. E. Felter; ORNL S. J. Pennycook; Drexel Univ. N. J. DiNardo

\section{$\underline{\text { Abstract }}$}

The goal of this project is to closely couple a multi-disciplinary materials research program with state-of-the-art advances in software and hardware at Sandia to develop a fundamental understanding of covalently-bonded disordered thin film materials. These materials are amorphousto-nanocrystalline structures made from light atomic weight elements $(B, C$, and $N)$. We have initiated our work through applications-focused laboratory directed R\&D (LDRD) and industrial CRADA programs. These efforts, while successful, have demonstrated the overwhelming need for an in-depth conceptual understanding of the relationship between growth energetics, atomic structures and materials properties. We will use first-principles theory, density-functional electronic structure geometry optimizations, to determine representative atomic configurations. This will allow direct comparison with experimental measurements, such as vibrational and optical spectroscopies, and radial distribution functions. The ability to relate theory with experiment serves both to validate the theory and to provide physical interpretations to the spectroscopies. A further goal is to gain an understanding of the relationship between the growth energetics and the resulting thin film structures, resulting in the ability to design engineered materials for specific applications.

\section{Purpose}

The current and potential impact of covalently-bonded disordered thin films is enormous. They are used as electron emitters for switch-tube components (DP-weapons technology) and are under development as active elements for both environmentally-friendly alternatives to fluorescent light bulbs and novel low-power, high-visibility flat-panel display technologies, as encapsulants for both environmental protection and microelectronics, as optical coatings for laser windows, and as ultrahard tribological coatings for integrated micro-electro-mechanical machines (IMEMs) as well as for machine tools. Disordered bonding networks allow conformal coatings that are ultra-hard and chemically-resistant on most surfaces. These materials can be deposited using energetic growth conditions, such as high-energy pulsed-laser deposition. Growth energetics control and determine the resulting bonding and nanostructures. Materials properties such as electrical conductivity, permittivity, optical absorption, and hardness are each tunable over a wide range. The multiplicity of metastable bonding types that can occur for even a single element such as carbon, which offers the coexistence of disordered 3-fold and 4-fold coordinated bonds, makes these material systems difficult to handle theoretically. Nevertheless, state-of-the-art computational advances in software 
and hardware at Sandia closely coupled with a multi-disciplinary experimental research program makes fundamental understanding of these complex systems attainable.

Covalently-bonded disordered materials are widely used and yet poorly understood. This class of materials includes amorphous-to-nanocrystalline structures made from light atomic weight elements from the first row of the periodic table. In addition, these materials have compositions and geometries that differ from naturally occurring and thermodynamically stable compounds. Examples are amorphous tetrahedral diamond-like carbon (a-tC), carbon-nitride, boron-carbide and boron-carbon-nitride. It should be emphasized that this class does not include highly crystalline materials like diamond. In addition, we do not include hydrogen-stabilized materials such as typical diamond-like carbon (DLC). It should be noted that carbon will not form five-fold coordinated sites as does silicon in a-Si, which in turn does not form three-fold coordinated sites as in a-tC; i.e. a-tC is not analogous to a-Si.

Our work to date has demonstrated the overwhelming need for a fundamental understanding of the relationship between growth energetics, atomic structures and materials properties. While it has been possible to grow some of these materials and achieve some tailoring of their properties via growth energetics, their full potential has yet to be realized. Moreover, a fundamental understanding of the relationship between local bonding structures and measurable properties is nonexistent. The difficulty is due to the low-atomic weight and the lack of crystallinity in these materials. There presently exists a need for state-of-the-art theoretical calculations to determine representative atomic structures so as to be able to relate bonding structures to observable properties, such as vibrational and optical spectra, and radial distribution functions. The direct comparison of theory with experiment would both validate the theory and provide physical interpretations to the spectroscopies. To date, most of the work has centered on a-tC. Due to its compositional simplicity, we use it as a prototype system for first-principles theoretical modeling. Calculations on more complex materials systems, i.e. those with two or more elements, will become more tractable with anticipated hardware and software advances. Finally, development of a fundamental understanding of materials properties based upon knowledge of realistic amorphous-tonanocrystalline geometries should ultimately enable the design of engineered materials for specific applications.

\section{Budget}

We request $\$ 695 \mathrm{~K}$ for FY98 to start this research program. Approximately $20 \%$ will be for the materials growth effort, $20 \%$ for the theoretical effort, and $60 \%$ for materials characterization. The latter includes funds for the research to be performed at Oak Ridge National Laboratory and Drexel University. 


\section{Research Program Summaries}

\section{A. Significant Accomplishments}

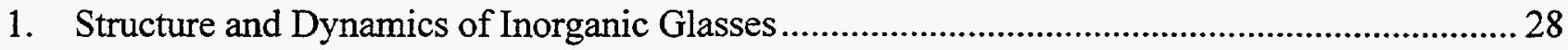

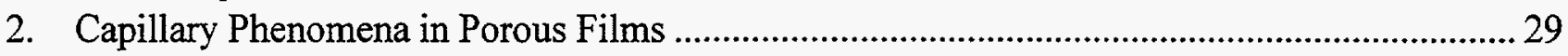

3. Direct Measurement and Atomistic Simulation of Molecular Mechanics ...................................30

4. Quantification and Understanding of Extreme Strengthening in Multiphase Thin Films.......... 31

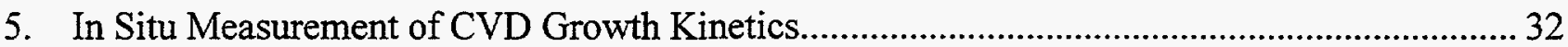

6. Controlled, Nanoscale Defects for Enhanced Critical Current Density in TlBaCaCuO Thin

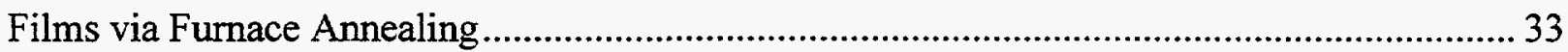

7. First Principles Model for Crack Embrittlement by Liquid Metals............................................. 34

8. Time-Resolved Photoinduced Absorption in $\left(\mathrm{Al}_{\mathrm{x}} \mathrm{Ga}_{1-\mathrm{x}}\right)_{0.5} \mathrm{In}_{0.5} \mathrm{P}$ Fractal Quantum Well Heterostructures

9. Direct Measurements of Si Surface Diffusion on Si(001) …....................................................... 36

10. Pair Breaking in Boron Carbides' Small-Bipolaron Hopping ...................................................... 37

11. Group V Inhibition of the GaAs and AlAs MOCVD Growth Rates ..........................................38

12. Semiconductor Nanoclusters as Potential Photocatalysts...............................................................39 


\section{Structure and Dynamics of Inorganic Glasses KC 020101}

\section{$\underline{\text { Scientific Accomplishment }}$}

We examined the molecular structure and time dependent relaxation processes of a mixed alkali $(\mathrm{Na}, \mathrm{Li})$ metaphosphate glass above and below the calorimetric glass transition temperature, $\mathrm{Tg}$. These studies provided insight into microscopic processes that control dynamic properties such as ionic conductivity, mechanical relaxations, nuclear spin relaxations and viscous flow.

Brillouin scattering studies of the glass in the liquid state indicate that the characteristic time of the response of the glass is comparable to that of viscous flow processes at that temperature. It is also evident that the nuclear spin relaxation rates exhibited by the ${ }^{31} \mathrm{P}$ nuclei are comparable. At low temperatures, the $\mathrm{Na}$ and $\mathrm{Li}$ nuclear spin relaxation rates are many orders of magnitude faster than that of ${ }^{31} \mathrm{P}$ whereas at temperatures far above $\mathrm{Tg}$ the time scales become comparable. These findings clearly indicate a coupling of relaxation modes as the temperature increases.

\section{Significance}

To date there is virtually no understanding of the microscopic processes that control the viscous flow of glasses. One proposal by Stebbins et al. makes an attempt at describing such a process in silicates where atomic ( $\mathrm{Si}$ ) entities appear to undergo time-dependent coordination and orientation changes to accommodate flow. This model, which first appeared in Nature, in addition to being not well accepted, is wholly inadequate. For example it can't possibly account for the dynamic behavior of modifying alkali cations. Our results from Brillouin scattering, NMR and viscous flow measurements provide the first clues into the design of new experiments to elucidate the microscopic mechanisms of viscous flow in inorganic glasses. A mechanistic understanding of this process would have far-reaching consequences in the US glass industry, by providing a rationale for the processing conditions of all glass compositions.

Performers: $\quad$ R.K. Brow, P.F. Green and D.L. Sidebottom, and J.J. Hudgens 


\section{Capillary Phenomena in Porous Films \\ KC 020101}

\section{$\underline{\text { Scientific Accomplishment }}$}

We observed that for liquids (e.g. water, methanol, acetonitrile) contained in pores less than $0.7 \mathrm{~nm}$ in diameter, the pores remained full (saturated) to relative pressures of about $10^{-3}$. Over the relative pressure range 1 to $10^{-3}$, the capillary tension scaled as $R T / V_{m} \ln \left(P / P_{o}\right)$ (where $V_{m}$ is the molar volume) in keeping with the expectations of bulk thermodynamics despite the fact that the liquid was confined to pores only one molecule wide. For methanol a tension of $380 \mathrm{MPa}(3.8 \mathrm{Kbar})$ was developed at $P / P_{O}=10^{-3}$. This compares with a tension of about $25 \mathrm{MPa}$ needed to cavitate bulk methanol. The implication is that when the pore size is less than the critical nucleus size, cavitation is avoided enabling the development of extremely high drying stresses.

\section{Significance}

High drying stress enables a "solvent templating" technique to precisely control film pore size in the range $0.4-1.0 \mathrm{~nm}$ of interest for gas separation. We demonstrated that when weak nascent networks were subjected to high capillary stress, the resulting pore size equaled the molecular size of the pore fluid molecule, e.g. water, methanol, ethanol, etc. The implication is that we can now "tune" the pore size for applications in membranes, sensors, and adsorbents.

This templating technique can be turned around as a sensitive new means of characterizing the pore size of microporous and mesoporous films. Beginning with a stressed, partially saturated film, we expose the film to a series of molecular probes of decreasing size. For molecules larger than the pore size, no stress relaxation is observed. For molecules much smaller than the pore size, stress relaxation is immediate, and for molecules approximately equal to the pore size, stress relaxation is characterized by a time constant of the order of hours.

Performers: J. Samuel, C.J. Brinker, and A.J. Hurd 


\section{Direct Measurement and Atomistic Simulation of Molecular Mechanics KC 020102}

Scientific Accomplishment

We have made the first ever measurement of the elastic modulus of a single monolayer of diacetylene molecules using acoustic wave damping. This novel technique measures the elastic energy storage and dissipative loss within a molecular film chemically bonded to an electrode of a high-frequency quartz crystal resonator undergoing periodic shear deformation. Di-acetylene-thiol molecules in solution will self-assemble onto a gold electrode surface to form a robust, twodimensionally ordered array of nearly vertically aligned molecules. The elastic modulus of this monolayer, $0.4-1.2 \mathrm{MPa}$ for shear rates of $\sim 0.05-1.0 \mathrm{~m} / \mathrm{sec}$, was determined from a multi-frequency analysis of the acoustic response data. Because this technique does not involve contacting the molecular layer with a probe to induce elastic deformation, modeling of the film dynamics is simplified. At the highest shear velocities, the molecular deformations occur on a time scale that can be accurately modeled by molecular dynamics simulations. Initial results for a perfectly ordered alkane-thiol monolayer confirm our experimental finding of no measurable energy dissipation within the film and predict an alkane chain-length dependence in the elastic modulus. The direct comparison of macroscopic measurements with molecular dynamics simulations enables us to relate the ensemble mechanical properties to atomistic potentials.

\section{Significance}

Self-assembled molecular monolayers are good models for studying the viscoelastic properties of molecular films and have demonstrated potential as lubricant layers in micro-machine sensor and actuator devices. The combination of a non-destructive experimental probe and realistic simulations using accurate molecular potentials will enable us to predict and verify the individual effects of molecular structure, intermolecular bonding, two-dimensional order, and impurity incorporation on the mechanics of a lubricating film. An understanding of how the variations in microscopic structure and bonding are manifested in macroscopic film performance is essential for successful film engineering at sub-micron dimensions. Most importantly, our combination of atomic-scale viscoelastic measurements and molecular dynamics simulations provides a new opportunity to understand a wide variety of materials phenomena, including the interfacial wetting, flow and creep of technologically relevant metal and ceramic systems.

Performers: N. D. Shinn and T. A. Michalske, Sandia National Laboratories

U. Landman, Georgia Institute of Technology

T. Kim and R. Crooks, Texas A\&M University 


\section{Quantification and Understanding of Extreme Strengthening in Multiphase Thin Films KC 020103}

\section{$\underline{\text { Scientific Accomplishment }}$}

The quantitative measurement and fundamental understanding of micromechanical properties were brought to a new level in studies of submicrometer aluminum-oxygen alloy layers synthesized using ion implantation, pulsed laser deposition, and electron-cyclotron-resonance plasmas. The investigated alloys consisted of metallic Al containing a very high density of small, hard $\mathrm{Al}_{2} \mathrm{O}_{3}$ particles, typically $1 \mathrm{~nm}$ in size and separated by about $1 \mathrm{~nm}$; these particles strongly impede the movement of dislocation defects responsible for plastic yielding, thereby raising the strength of the layers into the range of hard steels.

The quantitative measurement of micromechanical properties was accomplished by diamond-tip indentation coupled with finite-element computer modeling of the observed deformation to extract the fundamental properties of interest. Our earlier, pioneering work in this area was extended in two important respects: first, application of an improved computer code facilitated extensive theoretical modeling of experimental results using not only the conventional two-dimensional approximation but also a more rigorous treatment in three dimensions; additionally, the information content of indentation data was substantially enhanced by using rigid substrates beneath the alloy layers and by detailed characterization of the indentation tip. With these refinements, plastic yield strengths ranging from 0.04 to $5 \mathrm{GPa}$ were quantitatively determined together with the associated Young's elastic moduli. The method was validated by using the computer model with independent information to predict the measured force-versus-penetration curve for indentation of pure, annealed $\mathrm{Al}$; prediction and measurement agreed to within $5 \%$.

Fundamental understanding of the mechanical properties was achieved by applying the theory of dislocation motion within a metal matrix containing impenetrable inclusions. The measured plastic yield strengths, which ranged over two orders of magnitude among the alloys, were explained quantitatively to within a factor of two, even at the extreme and heretofore unattained levels above $1 \mathrm{GPa}$. The elastic response at small deformations, which does not involve dislocation motion, is predicted to change only slightly; in fact, the measured Young's elastic moduli varied by less than a factor of two.

\section{Significance}

Submicrometer hard layers formed by nonthermal methods are proving to be highly effective in reducing friction and wear in applications ranging from machine bearings to information-storage media. Continuing advances depend importantly on the ability to measure fundamental mechanical properties of the films. Additionally, efficient progress requires a mechanistic understanding of the relationship between mechanical properties and the film microstructure, which can then be manipulated through processing. These two goals have proved elusive because the layers of interest are microscopic, are coupled to substrates with very different mechanical properties, and have complex microstructures with feature sizes extending below $1 \mathrm{~nm}$. The present results represent important progress toward both goals. Moreover, the methods and insights developed here are believed widely applicable to multiphase hard layers.

\section{Performers: $\quad$ J.A. Knapp, J.C. Barbour, D.M. Follstaedt and S.M. Myers}




\section{In Situ Measurement of CVD Growth Kinetics \\ KC 020103}

Scientific Accomplishment

Using in situ X-ray reflectivity, we have measured the evolution of thin films of $\mathrm{Fe}$ on $\mathrm{Si}(001)$ substrates during chemical vapor deposition (CVD). The development of thin film structure during CVD growth has been difficult to study because the high ambient growth pressures prevent the use of standard electron-based surface diagnostics. The X-ray reflectivity technique allows us to simultaneously measure the change in film thickness and surface roughness during CVD growth. These measurements have allowed us to clearly identify and separate the nucleation regime from the continuous growth regime. Using classical nucleation theory, we have shown that the precursor molecule $\left(\mathrm{Fe}(\mathrm{CO})_{5}\right)$ preferentially decomposes on the $\mathrm{Fe}$ surface rather than the Si surface and obtained activation energies for these processes. A decrease in the surface roughness after the initial nucleation regime enables us to determine when the individual nuclei coalesce to form a continuous film during processing. Unlike molecular beam epitaxy (MBE) where all the deposited atoms stick to the surface, the interaction of the chemical precursor molecule with the surface greatly complicates the CVD growth process. These experiments have enabled us to determine that the density of $\mathrm{Fe}$ nuclei is higher at high temperatures (the opposite result from MBE) in agreement with STM studies of the surface.

\section{Significance}

CVD is a very important process for thin film deposition, but the kinetics of thin film evolution have been difficult to study because of the high processing pressure. Advancing CVD science relies on the development of in situ diagnostics for fuller characterization of the growth process. Our studies demonstrate the feasibility of using X-ray reflectivity to study the evolution of the surface and film structure in real-time. Important kinetic parameters such as the growth rate can be obtained non-destructively from a single growth sequence. In addition, the highly non-linear growth kinetics in the early stages of film formation can be studied directly to determine the nucleation mechanisms.

Performers: B.K. Kellerman and E. Chason

(BKK was also a graduate student at the University of Texas, Austin) 


\section{Controlled, Nanoscale Defects for Enhanced Critical Current Density in TIBaCaCuO Thin Films via Furnace Annealing KC 020202}

\section{$\underline{\text { Scientific Accomplishment }}$}

Most applications of high temperature superconducting (HTS) thin films require large current densities in strong magnetic fields at liquid nitrogen temperatures. Under these operating conditions, the magnetic field penetrates the film in discrete, quantized flux bundles called magnetic vortices. The vortices move due to the strong Lorentz force (current density $\times$ magnetic field) and the significant amount of thermal energy. This motion causes energy dissipation and limits the operating current density. The vortices can be pinned at nanoscale defective regions where the superconductivity is weakened. We have shown that simple furnace annealing at $600^{\circ} \mathrm{C}$ of $\mathrm{TlBa}_{2} \mathrm{CaCu}_{2} \mathrm{O}_{\mathrm{x}}(\mathrm{Tl}-1212)$ thin films creates such defects in the microstructure. We have also shown that this annealing produces substantially increased vortex pinning and, consequently, a large increase in the observed critical current density at elevated temperatures in strong magnetic fields. The nanoscale defects are likely to be the origin of the enhanced vortex pinning. Prior studies by us and others have used high-energy ion or neutron irradiation to create defects and increase the vortex pinning.

Using high-resolution transmission electron microscopy (HRTEM), we have explicitly identified nmsized defects in the microstructure of the annealed films. Prior to the post-anneal, as-grown films exhibit near-perfect crystallinity. Following the post-anneal, the films contain numerous stacking faults that result in nm-sized discontinuities in the atomic layers. Given the density, size and orientation of these faults, it is plausible to expect enhanced vortex pinning. Magnetic relaxation data show a significant increase, for annealed relative to as-grown films, in the effective vortex pinning potential $U_{\text {eff }}(J, T)$ where $J$ is the current density and $T$ the temperature. Higher $U_{\text {eff }}$ translates directly into lower dissipation at a fixed operating condition or, equivalently, a substantially. higher $\mathrm{J}$ at constant dissipation. In other words, such superconducting films can be operated at higher temperatures in stronger magnetic fields.

\section{Significance}

We have used simple furnace annealing to generate nanoscale defects that correlate with substantially enhanced vortex pinning and a large increase in the critical current density for Tl-1212 HTS films. Improving vortex pinning is necessary for most applications of HTS materials. Among the most promising applications for HTS films are microwave device components such as oscillators, filters, delay lines and antennas. In such applications $\mathrm{TlBaCaCuO}$ films excel relative to other HTS films because of their comparatively low microwave surface resistance and high power handling capability. This already enables substantial reductions in both the size and weight for microwave components. Increasing the operating current density by introducing and controlling the defects should permit still higher microwave power capability, bringing this technology closer to reality. Correlating defect structures with enhanced pinning potentials will provide insight into the rich physics of the complex phenomena underlying this technology.

Performers: P. P. Newcomer, E. L. Venturini and M. P. Siegal 


\section{First Principles Model for Crack Embrittlement by Liquid Metals KC 020102}

\section{$\underline{\text { Scientific Accomplishment }}$}

We have performed first principles calculations of the energetics and structure of ultrathin Ga layers on $\mathrm{Al}(111),(100)$ and (110) surfaces. Consistent with a thermodynamic interpretation of the observation that $\mathrm{Ga}$ causes single-crystal Al to crack on (100) planes, we find that: 1) Ga binds more strongly to Al than to itself, i.e. Ga wets $\mathrm{Al}$ surfaces, and 2) the Ga-covered (100) plane has the lowest surface energy, representing a compromise between weaker $\mathrm{Ga}$ binding on closer packed $\mathrm{Al}$ (111) surface and higher clean surface surface-energies on more open $\mathrm{Al}$ (110). In the second adsorbed $\mathrm{Ga}$ layer, on $\mathrm{Al}(100)$, we find several low energy structures, including a weakly dimerized adatom arrangement. The propensity for $\mathrm{Ga}$ to dimerize may explain why the strongly adsorbed $\mathrm{Ga}$ doesn't simply glue the crack together by bridging across the Al crack opening. We attribute Ga's ability to form many low energy adsorption structures to the rather high $\mathrm{Ga}(4 \mathrm{~s}->4 \mathrm{p})$ promotion energy. The existence of these structures is consistent with low diffusion barriers and thus fast $\mathrm{Ga}$ migration to crack tips at room temperature.

\section{Significance}

The embrittlement of metals by chemical agents has a significant impact on our ability to predict the reliability of engineering structures, particularly those exposed to aggressive chemical environments. Metal embrittlement by liquid metals is an important example of this type of chemical/mechanical effect of which the embrittlement of $\mathrm{Al}$ is of particular technological significance. Previous fracture simulations have relied on empirical bonding potentials that can't address the chemical basis for the metal embrittlement phenomenon. Our new theory that is based on first principle calculations is able to provide the relevant thermodynamic and structural parameters needed to model the role on chemistry on the embrittlement process. The results suggest that the effectiveness of $\mathrm{Ga}$ as an embrittling agent is due to the high valance state promotion energy for $\mathrm{Ga}$ which prevents it from forming many bonds and therefore precludes the formation of a bridge across the crack tip. This represents the first model to address liquid metal embrittlement from a chemical view and provides a new approach to predicting embrittlement effects

Performers: P. J. Feibelman and R. R. Stumpf 


\section{Time-Resolved Photoinduced Absorption in $\left(\mathrm{Al}_{\mathbf{x}} \mathbf{G a}_{1-\mathrm{x}}\right)_{0.5} \mathbf{I n}_{0.5} \mathrm{P}$ \\ Fractal Quantum Well Heterostructures \\ KC 020105}

\section{$\underline{\text { Scientific Accomplishment }}$}

We have measured the relaxation processes of photoinjected carriers in $\left(\mathrm{Al}_{\mathrm{x}} \mathrm{Ga}_{1-\mathrm{x}}\right)_{0.5} \mathrm{In}_{0.5} \mathrm{P}$ fractal quantum well heterostructures (FQWHs) using femtosecond photoinduced absorption. In these measurements, carriers were photoinjected near the surface of the sample using a short $(\sim 150 \mathrm{fs})$ optical pulse, and the time-evolution of the changes in the absorption spectra of the samples was recorded using a broadband probe pulse. The differential absorption spectra recorded in this manner allow the determination of the distribution of photoexcited carriers within the structure, since the absorption strength of a quantum well populated by carriers is reduced due to state filling effects. A sequence of four FQWHs were investigated with characteristic well/barrier widths of $50 \AA, 100 \AA$, $200 \AA$ and $400 \AA$. The results of the photoinduced absorption measurements show that photoinjected carriers initially occupy the higher-lying quantum wells located near the surface of the sample where they were injected. With increasing time, the carriers filter through the structure and populate the lower energy quantum wells. At the longest delay times, the carriers reach the lowest lying well in the center of the structure, where they remain until they recombine. As the characteristic width of the wells and barriers increases, the time required for the carriers to reach the central well increases from 100 ps to about 1 ns.

\section{Significance}

Semiconductor light sources are used widely in displays, communications, and analytical systems. Nearly all of these emit light in narrow spectral bands. Broadband spectral emitters are of great interest for illumination sources, but have not been developed. Fractal structures have shown spectrally-broadband emission and may be used to realize such light emitters. The spectral breadth of luminescence from these novel semiconductors is determined by dwell time of carriers in higherlying quantum states. If this time is comparable to the radiative lifetime, then a considerable luminescence can be expected at higher photon energies. The results of the photoinduced absorption show that this dwell time can be simply controlled by changing the characteristic width of the wells and barriers. These findings are confirmed by luminescence measurements which show that the emission at higher photon energies increases drastically with the characteristic well/barrier width. The information gained in these investigations is vital to design of efficient, broadband emitters.

Performers: M. B. Sinclair, M. H. Crawford, and P. L. Gourley 


\section{Direct Measurements of Si Surface Diffusion on Si(001) \\ KC 020202}

$\underline{\text { Scientific Accomplishment }}$

We have used Sandia's newly developed "atom-tracker" scanning tunneling microscope (STM) to investigate the movements of individual dimers (two-atom clusters) on the (001) crystal plane of silicon. We find that in the temperature range between 25-130 $\mathrm{C}$ the average hop rate of dimers increases by four orders of magnitude -- from just several hops per hour to 10 hops per second. Quantitative measurements of hop rates over this extensive range are not possible with conventional STMs, but can be achieved with the 1000-fold increase in time resolution offered by the atomtracker. Unlike ordinary STMs, the probe tip of the atom tracker is locked onto a selected atom or cluster using lateral feedback. When a diffusion event occurs the atom tracker quickly relocates to the species new position. Because every diffusion event is resolved, we can measure the statistics of the diffusion path -- we do not need to assume random walk statistics. In the case of Si dimers on $\mathrm{Si}(001)$, the hop statistics show that away from surface defects the displacement rate follows the expected Arrhenius relation (the log of the hop rate is linear with inverse temperature), but near surface defects the diffusion process is measurably altered. Detailed analysis of the dimers movements show that the mass-transport pathways are quite complicated involving anisotropic cluster migration and atom-defect interactions that dominate the long-range diffusion process.

\section{Significance}

Understanding the dynamical behavior of atoms and small clusters on semiconductor surfaces is a key element in developing a science-based approach to the fabrication of microelectronic devices. By monitoring the details of the diffusion path of selected atoms, the atom-tracker allows us to measure individual kinetic processes on the atomic scale, as well as the energetics of atom-atom, atom-defect, and atom-substrate interactions.. Adopting the view of the "atom as a probe" opens up many new areas of investigation into materials surface processes. From observations of atom diffusion over heterogeneous surfaces, we will be able to obtain a map of the surface composition on materials where there is no contrast mechanism in conventional STM techniques. We will also be able to investigate the atomic-scale details of surfactant-mediated growth. Understanding surfactant-atom diffusion and ultimate incorporation in surfaces, and the energetics between surfactant and substrate atoms will lead to better control over growth morphology and device performance. By increasing the capability of scanning probe microscopies to yield quantitative measurements of surface kinetic processes that were previously inaccessible, we will provide additional insight into the complicated interplay between the processes at work during growth and etching.

Performer: B. S. Swartzentruber 


\section{Pair Breaking in Boron Carbides' Small-Bipolaron Hopping KC 020205}

\section{$\underline{\text { Scientific Accomplishment }}$}

The high-temperature hopping motion of the two electronic carriers that are bound together as a singlet small-bipolaron has been calculated. The hop rate of individual separated carriers is always sufficiently large compared with that for the collateral hopping of singlet pairs that the motion of broken pairs dominates small-bipolarons' dc conductivity. Thus, a significant contribution to the temperature dependence of small-bipolarons' dc conductivity arises from the thermally induced breaking of singlet pairs. The generation of spins expected from the dissociation of boron carbides' singlet small bipolarons is seen from $100 \mathrm{~K}$ to $1000 \mathrm{~K}$ in our ESR measurements.

\section{Significance}

Charge carriers are believed to form singlet small bipolarons in various transition-metal oxides and borides. Boron carbides are prototype systems for studying small-bipolaron hopping. Our work indicates that small-bipolaron hopping motion should always be associated with pair breaking. Our measurements on boron carbides demonstrate that the pair breaking can be observed with magnetic measurements.

Performers: D. Emin and T. Aselage in collaboration with O. Chauvet, L. Forro, L. Zuppiroli, Ecole Polytechnique Federale de Lausanne, Switzerland. 


\section{Group V Inhibition of the GaAs and AIAs MOCVD Growth Rates \\ KC 020303}

\section{$\underline{\text { Scientific Accomplishment }}$}

We have determined that high group V partial pressures inhibit the growth rate of GaAs and AlAs. This discovery was made possible through accurate growth rate measurements in a well characterized rotating disk MOCVD reactor. The data were obtained using an in situ reflectance monitor to measure growth rates of GaAs and AlAs from TMGa, TMAl, $\mathrm{AsH}_{3}$, and $\mathrm{tBuAs}$ precursors as a function of the V to III ratio. We observed that as the V to III ratio is increased by increasing the group $\mathrm{V}$ partial pressure, the growth rate decreases by 20 to $50 \%$ depending upon the reactants. We are able to explain these results with a new surface mechanism wherein As atoms occupy group III lattice sites on the surface partially creating the $c(4 \times 4)$ surface reconstruction and blocking group III surface reactions. Rate constants for the rate of desorption of these As surface atoms are in agreement with TPD measurements by Banse and Creighton.

The blocking mechanism has been tested against an extensive parameter space of data at $640^{\circ} \mathrm{C}$ for four combinations of group III and group V precursors. The tBuAs systems have a higher incorporation rate for As containing species into the $\mathrm{c}(4 \times 4)$ layer than their $\mathrm{AsH}_{3}$ counterparts, due to tBuAs's lower pyrolysis temperature. While the $640^{\circ} \mathrm{C}$ data is well represented by the model, experimental data at lower temperatures on the group $\mathrm{V}$ dependence demonstrates a complex "rollover" behavior, with growth rate increasing with group V pressure at low group V pressure. This suggests that the $c(4 \times 4)$ As does not form under these conditions, and that As supply is in some way deficient.

\section{Significance}

The group $\mathrm{V}$ inhibition effect has important ramifications for our fundamental understanding of the types of surface ligands present during normal MOCVD growth. In the absence of accurate growth rate data, the phenomenon had not been previously observed in MOCVD, although a similar phenomenon has been reported in metal-organic MBE where the overall surface kinetics are not obscured by mass transport limitations. The observed blocking mechanism is not a part of previously published detailed kinetics models of III-V growth behavior, necessitating a reexamination of these earlier mechanisms.

Performers: H. K. Moffat, K. P. Killeen and K. C. Baucom 


\section{Semiconductor Nanoclusters as Potential Photocatalysts \\ KC 020303}

\section{$\underline{\text { Scientific Accomplishment }}$}

Using our patented inverse micellar synthesis approach, we have successfully grown high quality, crystalline, monodisperse nanometer-size (or nanoclusters) of $\mathrm{PbS}, \mathrm{FeS}_{2}, \mathrm{MoS}_{2}, \mathrm{CdS}$ and $\mathrm{WSe}_{2}$ and demonstrated optical properties that make them attractive candidates as photocatalysts for solar fuel production and solar detoxification. In such applications these semiconductors would absorb light creating electron-hole pairs and thus could catalyze specific chemical reactions. To be effective as a solar photocatalyst, the semiconductor must have a bandgap that is matched to the solar spectrum, and the energies of the valence and conduction band edges must be compatible with the oxidation and reduction potentials for the reactions involved. Additionally, the material must be resistant to photochemical degradation, and the carrier diffusion time to the surface must be shorter than the electron-hole recombination time. Semiconductor nanoclusters are in principle ideally suited to meet these requirements. Their small size allows fast carrier diffusion to the surface, and they possess the ability to continuously tune the bandgap (by up to $1 \mathrm{eV}$ or more) by changing cluster size as a consequence of quantum confinement of the charge carriers.

We have shown that the bandgaps of the above semiconductors, which are typically in the mid to near infrared in the bulk, can be shifted to the near ultraviolet by reducing cluster size. Using PbS as a model system, we have demonstrated that most of the absorbed light energy is channeled into a non-radiative process. We observed surface-related emission but little bulk recombination, features that bode well for potential applications of these nanoclusters in photocatalysis. Depending on the sign of the change in charge state of the surface recombination sites (or traps), the excited nanoclusters are capable of initiating either reduction (electron trap) or oxidation (hole trap) reactions. Results on gold capping the nanoclusters show that surface related states of one material can be excited through states of a different interior material. This effect has extremely important ramifications for photocatalysts since the functions of energy absorption and energy transfer can be separated. Materials for the interior of the nanocluster can be chosen to be efficient absorbers of light energy, while materials on the surface can be chosen to be efficient at transferring energy to reactants.

\section{Significance}

This research has demonstrated significant progress toward the ultimate goal of using nanoclusters as efficient photocatalysts. The ability to successfully cap the clusters with metals is an important step. The specific direction of our work was guided by a practical consideration -- the need for inexpensive, disposable photocatalysts; hence our emphasis on sulfides. $\mathrm{MoS}_{2}$ is particularly attractive because of its known stability (in bulk form) in photoelectrochemical environments. More work is needed to develop these materials and apply them for such important reactions as the solar decomposition of $\mathrm{H}_{2} \mathrm{O}$ and $\mathrm{H}_{2} \mathrm{~S}$ and the solar decomposition of chlorinated hydrocarbons in water.

Performers: J. P. Wilcoxon, D. E. Bliss, P. P. Newcomer, and G. A. Samara 
This page intentionally left blank. 


\section{Research Program Summaries}

\section{B. Research Summaries}

1. Physics and Chemistry of Ceramics

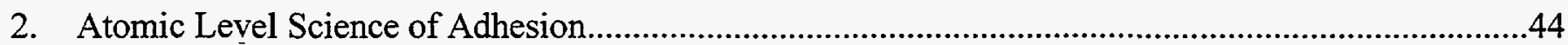

3. Wetting and Flow of Liquid Metals and Amorphous Ceramics at Solid Interfaces ...............................46

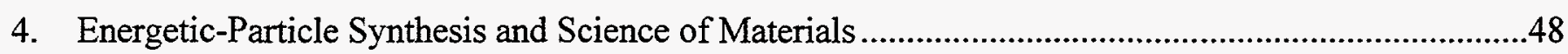

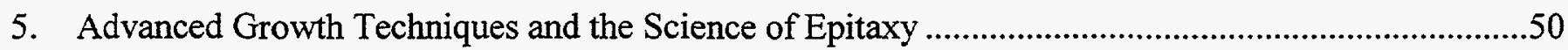

6. Artificially-Structured Semiconductor Materials Science …................................................................

7. Physics and Chemistry of Novel Superconductors …….................................................................54

8. Tailored Surfaces and Interfaces for Materials Applications ..............................................................56

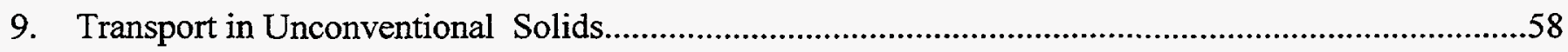

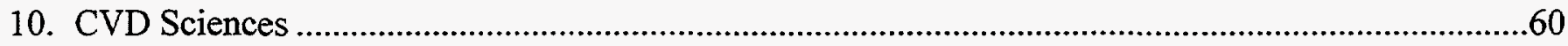

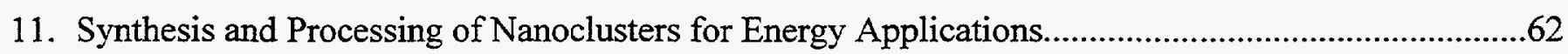

12. Field-Structured Anisotropic Composites.......................................................................................64 


\section{Physics and Chemistry of Ceramics \\ KC 020101}

Principal Investigators: R. A. Assink, T. J. Boyle, C. J. Brinker, R. K. Brow, R. A. Cairncross, K. Chen, P. F. Green, J.J. Hudgens, A. J. Hurd, J. Samuel, P. R. Schunk, R. W. Schwartz, D.L. Sidebottom

Goals: To understand the chemical and physical processes that determine the structure and properties of ceramics and glasses and to establish a fundamental technology base for DP ceramics.

\section{Recent Highlights:}

- Brillouin scattering from molten glass showed viscosity-controlled relaxations that compare with spin relaxation rates of ${ }^{31} \mathrm{P}$ nuclei. At low temperatures, $\mathrm{Na}$ and $\mathrm{Li}$ nuclei relax much faster than ${ }^{31} \mathrm{P}$ whereas in the melt the time scales are comparable, suggesting increased coupling between relaxation modes.

- Overexpanded methanol in micropores, created by templating the film around the solvent molecule itself, exhibited a tension of 3.8 Kbar (sic), though it cavitates at only $0.25 \mathrm{Kbar}$ in bulk: Cavitation is suppressed when the pore size is less than a critical nucleus.

- Transport in deformable porous media and aerogel "springback" were described in a numerical theory in which the pores and moduli change self consistently, with key insights from an analytical model as well.

- Cross-condensation reaction rates: Developed an experimental procedure to detect the exchange of alkoxy and hydroxyl groups in acid catalyzed silicate sols. The equilibrium exchange directly impacts the relative formation rates of self- and cross-condensed species.

- Multi-nuclear NMR of ${ }^{17} \mathrm{O}$ and ${ }^{7} \mathrm{Li}$ ligands along with single crystal XRD of products in titania and other sols were used to understand fourth order reactivity and analogous behavior in the PZT system.

- Ligand effect on film processing was established between pyrolysis temperature and crystallization of films using thermodynamics and standard nucleation and growth theory.

- Statistical processes in ceramic powder compaction. Ceramic powder compaction was successfully described by particle rearrangement then a novel Boltzmann-activated granular fracture.

\section{Future Directions:}

- Mechanistic understanding of glass flow. Stebbins et al. in Nature ascribe silicate flow to Si recoordination and reorientation, but can't account for the dynamics of alkali cations. We will develop a theory based on anomalous diffusion that promises profound consequences in the US glass industry.

- Find and elucidate the elusive gel point during film deposition by three strategies: viscoeleastic response of the depositing film by a glaucoma test (jet of air), rotational diffusion of fluorescent groups tethered to the network, and a numerical theory of gelation that utilizes a framework with an evolving gel structure.

- Develop novel catalytic, conductive, and bio-active aerogels e.g. B-doped for neutron capture therapy.

- Stress isotherms of porous thin films will be studied by dipcoating in a chamber under a controlled atmosphere while measuring stress, thickness, index, and mass change. Of interest are the large overshoots in tension, when the overlying pressure is reduced, and the anomalous behavior of water.

- Densification of thin films will be studied using steric hindering acetates, amides, and nitrogen-containing ligands, as well as the effects $M-N$ versus $M-O$ linkages have on the final film characteristics.

- Multi-nuclear NMR Characterization Techniques. ${ }^{17} \mathrm{O}$ and ${ }^{15} \mathrm{~N}$ (new) and 2D NMR will be used to investigate the formation, products, and kinetics of titanium sol-gels.

- Stress-isotherm measurements of INEL and LLNL materials. The pore size distributions and adsorption behavior of resourcinolformaldehyde (RF) aerogels (LLNL) and nonporous membranes (INEL) will be studied by our new method of adsorption-induced stress. (S\&P Center)

- Modeling of LLNL RF aerogel drying and adsorption. Our code developed for aerogel films will be adapted to RF aerogels made by the Livermore process. (S\&P Center)

- Thermal conductivity methodology study for porous films. An experimental and theoretical strategy will be developed jointly by SNL, LLNL, and INEL, in consultation with Jochen Fricke (Stuttgart) to answer the mutual need to measure the thermal conductivity of thin films. (S\&P Center)

- Joining technologies with Center of Excellence. A workshop will be held to discuss recent developments in the joining of ceramics and other materials. (S\&P Center)

Relationship to DOE Technologies: Our BES technology base now supports studies in aging and reliability for Defense Programs, silica- reinforced silicones for the Dow Corning TTI CRADA, METC-funded separation membranes, numerous internal LDRD projects, and SmartProcess of multi-component thin films and ceramic powder compaction for ADaPT. NonDOE interactions include ARPA ferroelectric films and novel PZT processing for Metatek.

Program Quality: 9 invited presentations and publications, 2 patents filed, Iler Award for Colloid Chemistry and the BES Sustained Outstanding Research Award for Metallurgy and Ceramics. 


\section{Physics and Chemistry of Ceramics \\ KC 020101}

\section{Publications}

\section{Work Performed Under Present BES Program}

1. "NMR Investigation of the Structures of Phosphate and Phosphate-Containing Glasses: A Review," R. J. Kirkpatrick and R. K. Brow, Solid State Nuclear Magnetic Resonance 5, 9-21 (1995).

2. "Comparison of KWW and Power Law Analyses of an Ion-Conducting Glass," D.L. Sidebottom, P.F. Green, and R.K. Brow, J. Non-Cryst. Solids 183, 151 (1995).

3. "Anomalous Diffusion Model of Ionic Transport in Oxide Glasses," D.L. Sidebottom, P.F. Green, and R.K. Brow, Phys. Rev. B 51, 2770 (1995).

4. "Two Contributions to the ac Conductivity of Alkali Oxide Glasses," D.L. Sidebottom, P.F. Green, and R.K. Brow, Phys. Rev. Lett. 74, 5068 (1995).

5. "Metrical Comparison and Alkali Metal NMR Spectroscopic Investigation of a Congener Set of Alkali Metallated Titanium (IV) Iso-propoxides: X-ray Structures of [NaTi(OCHMe2)5]_and [KTi(OCHMe2)5]_" T. J. Boyle, D. C. Bradley, M. J. Hampden-Smith, A. Patel, and J. W. Ziller, Inorganic Chemistry 34, 5893 (1995).

6. "Synthesis, and Structure of Novel Group (IV) Tridentate Alkoxide Complexes and the Thin Film Derived Therefrom. $X$-ray Structure of: $\left(\mathrm{H}_{3} \mathrm{CC}\left(\mathrm{CH}_{2} \mathrm{~m}_{3}-\mathrm{O}\right)\left(\mathrm{CH}_{2} \mathrm{~m}-\mathrm{O}\right)_{2}\right)_{2} \mathrm{Ti}_{4}\left(\mathrm{OCH}\left(\mathrm{CH}_{3}\right)_{2}\right)_{10}, \quad\left(\mathrm{H}_{3} \mathrm{CCH}_{2} \mathrm{C}\left(\mathrm{CH}_{2} \mathrm{~m}_{3}-\mathrm{O}\right)\left(\mathrm{CH}_{2} \mathrm{~m}\right.\right.$ $\left.\mathrm{O}_{2}\right)_{2} \mathrm{Ti}_{4}\left(\mathrm{OCH}\left(\mathrm{CH}_{3}\right)_{2}\right)_{10}$, and $\left.\left(\mathrm{H}_{3} \mathrm{CC}\left(\mathrm{CH}_{2} \mathrm{~m}-\mathrm{O}\right)_{3}\right)_{2} \mathrm{Zr}_{4}\left(\mathrm{~m}-\mathrm{OCH}\left(\mathrm{CH}_{3}\right)_{2}\right)_{2}\left(\mathrm{OCH}\left(\mathrm{CH}_{3}\right)_{2}\right)\right)^{\prime \prime} \mathrm{T}$. J. Boyle, R. W. Schwartz, R. J. Doedens, and J. W. Ziller Inorganic Chemistry 33, 1110 (1995).

7. "Control of Thin Film Processing Behavior Through Precursor Structural Modifications," R. W. Schwartz, J. A. Voigt, T. J. Boyle, T. A. Christenson, and C. D. Buchheit, Ceramic Engineering \& Science Proceedings 16, 1045 (1995).

8. "In-Situ Flourescence Probing of the Chemical Changes during Sol-Gel Thin Film Formation," F. Nishida, J. M. Mckiernan, B. Dunn, J. I. Zink, C. J. Brinker, and A. J. Hurd, J. Am. Ceram. Soc., 78, 1640-48 (1995)

9. "Free Meniscus Coating Processes," P. R. Schunk, A. J. Hurd, and C. J. Brinker, in The Fundamentals of Thin-Film Coating: Implications and Technological Relevances, eds., S. F. Kistler and P. M. Shweizer, Chapman and Hall (1996).

10. "Ambient Pressure Silica Aerogel Films," S.S. Prakash, C. J. Brinker, and A. J. Hurd, J. Non-Cryst. Solids, 190, 264275 (1995)

11. "Silica Aerogel Films at Ambient Pressure via Surface Derivatization and Reversible Drying Shrinkage," S.S. Prakash, C.J. Brinker, A.J. Hurd, and S.M. Rao, Nature, 374, 439-443 (1995).

12. "GOMA - A Full-Newton Finite Element Program for Free and Moving Boundary Problems with Coupled Fluid/Solid Momentum, Energy, Mass, and Chemical Species Transport: User's Guide", P.R. Schunk, P.A. Sackinger, R.R. Rao, K.S. Chen, and R.A. Cairncross, internal Sandia report (1995).

13. "Organic 'Template' Approach to Molecular Sieving Membranes," N. K. Raman and C. J. Brinker, J. Membrane Sci., $105,273-279$ (1995)

14. "Structure Property Relationships in Silica Membranes and Films," C. J. Brinker, N. K. Raman, R. Sehgal, M. N. Logan, T. L. Ward, S. Wallace, and R. A. Assink, Journal of Sol-Gel Science and Technology, 4 [2] 117-133 (1995).

15. "Sol-Gel Derived Ceramic Films: Fundamentals and Applications", C.J. Brinker, A.J. Hurd, P.R. Schunk, C.S. Ashley, R.A. Cairncross, J. Samuel, K.S. Chen, C. Scotto, and R.A. Schwartz, in Metallurgical and Ceramic Coatings, edited by K.H. Stern, Chapman \& Hall, (1995).

\section{Work Performed Jointly with Other Programs}

16. "Alkylene-Bridged Polysilsesquioxane Aerogels: Highly Porous Hybrid Organic-Inorganic Materials," D. A. Loy, G. M. Jamison, B. M. Baugher, E. M. Russick, R. A. Assink, S. Prabakar and K. J. Shea, Journal of Non-Crystalline Solids 186, 44 (1995). 


\section{Atomic Level Science of Adhesion \\ KC 020102}

Principal Investigators: T. A. Michalske, J. E. Houston, N. D. Shinn, and P. J. Feibelman

Goals: To understand in atomic detail the nature of the physical and chemical interactions that control interfacial binding and to use this understanding to: 1) develop predictive models for adhesive bond stability and 2) tailor material surfaces and interfaces for optimized adhesion and lubrication properties.

\section{Recent Highlights:}

- Nanometer-Scale Mapping of Thin-Film Residual Stress - IFM/nanoindentation measurements on thin Au films deposited on various substrates showed considerable variation in the quantitative values for the elastic modulus and the maximum shear stress at plastic threshold. Through independent measurement of the thin film residual stress, we demonstrated that the variation in the measured nanoindenation response can be correlated with the sign and magnitude of the residual stress for the various films/substrate combinations. Our results indicate that IFM nanoindentation is the first experimental probe that can map thin-film residual stress with nanometer-scale spatial resolution. This development could have significant implication in the microelectronics industry where the ability to monitor and control residual stress in spatially patterned thin films is critical to eliminating deleterious processes such as delamination and stress voiding of IC interconnects.

- New Theoretical Model for Interfacial Crack Embrittlement - In order to understand adsorbate induced embrittlement we performed first principles calculations of the energetics and structure of ultrathin $\mathrm{Ga}$ layers on $\mathrm{Al}(111),(100)$ and (110) surfaces. Consistent with the experimental observation that Ga causes single-crystal Al to crack on (100) planes, we find that: 1) Ga binds more strongly to Al than to itself, i.e. Ga wets Al surfaces, and 2) the Ga-covered (100) plane has the lowest surface energy. The second adsorbed $\mathrm{Ga}$ layer on $\mathrm{Al}(100)$ shows a weakly dimerized adatom arrangement. We attribute Ga's ability to form many low energy adsorption structures to the rather high $\mathrm{Ga}(4 \mathrm{~s}->4 \mathrm{p})$ promotion energy. This structure is consistent with the low diffusion barriers and thus fast Ga migration to crack tips at room temperature.

- Plastic Threshold for Nanomechanical Contacts -Our previous measurements showed a marked increase in contact stress required to initiated plastic deformation with indenter probe radii below $400 \mathrm{~nm}$. We now show that this measured response is consistent with the results of a continuum-based model predicting an increased pressure for dislocation loop punching when the radius of the dislocation loop is less than 10 times the Burgers vector. This comparison suggests that the critical stress to stabilize a dislocation is controlled by long-range elastic interactions and not atomic-scale shear instabilities.

- Ultra-High Vacuum/IFM System - The newly completed UHV/IFM fitted with updated feedback control electronics has demonstrated continuous force versus displacement profiles for adhesive contact between clean metal surfaces. This new system provides the capability for sample and sensor interchange under vacuum, sputter cleaning and chemical surface analysis of both tip and sample. The new system also provides the ability to alter surfaces by gas dosing or metal evaporation. All previous nanomechanics and adhesive force measurements have been conducted in the presence of uncontrolled surface adsorbate layers on the probe tip as well as the sample surface. This new instrument will allow us to directly explore the role of surface adsorbates on adhesion and contact deformation by comparison with results for UHV clean conditions.

Future Directions: The program will continue to focus on nanometer-scale measurements of interfacial adhesion the development of theoretical models to address the chemical stability of the adhesive bond. The probe tips and substrate surfaces will be tailored with self-assembled monolayer films to controllably examine the role of surface chemical groups on the formation and stability of the adhesive bond. With the UHV/surface science IFM in operation, adhesion events will be examined on clean and adsorbate covered surfaces and the results compared with first principles atomistic simulations. We will also use the IFM to probe the mechanical response of small-scale structures so that we can better understand the behavior of materials near stress singularities and of nanoscale composite materials. We will also begin finite element and atomistic modelling to interpret nanoindentation results.

Interactions with DOE Technologies: Our fundamental studies of interfacial adhesion increase the reliability of composite materials interfaces that are important to DP funded R\&D and the Materials Joining Project of the DOE Synthesis and Processing Center.

Program Quality: 6 publications, 6 invited presentations (MRS, TMS, ACS, Adhesion Society) 


\section{Atomic Level Science of Adhesion \\ KC 020102}

\section{Publications}

1. Morphological and Nanomechanical Investigations of Surface Modified Poly(tetrafluoroethylene), A. J. Howard, R. R. Rye and J. E. Houston, J. Appl. Phys. (In Press).

2. Probing Adhesion Forces at the Molecular Scale, R. C. Thomas, J. E. Houston, R. M. Crooks, T. Kim and T. A. Michalske, J. Am. Chem. Soc. 117, 3830 (1995).

3. The Effect of Probe Radius on the Threshold Shear Stress for the Single-Crystal Au(111) Surface, J. E. Houston, T. A. Michalske, P. Tangjunyong, J. Mat. Res. (Submitted).

4. The Direct Observation of Inter-group bonding using Interfacial Force Microscopy, R. M. Crooks, R. C. Thomas, J. E. Houston, T. Kim and T. A. Michalske, J. Adhesion Soc. (In Press).

5. Growth and Oxidation of Ultra-Thin Al Films on Ru(0001), Y. Wu, H.-S. Tao, E. Garfunkel, T. E. Madey and N. D. Shinn, Surf. Sci. 336, 123 (1995).

6. Adsorption and Thermal Stability of Mn on TiO2 (110); X-Ray Absorption Spectroscopy and X-Ray Photoemission, U. Diebold and N. D. Shinn, Phys. Rev. B (in press). 


\section{Wetting and Flow of Liquid Metals and Amorphous Ceramics at Solid Interfaces KC 020102}

Principal Investigators: T. A. Michalske, N. D. Shinn, F. G. Yost, R. Rye, U. Landman (Georgia Tech.), R. Cannon (LBL)

Goals: To experimentally measure and theoretically model the atomic-scale processes that control fluid flow at solid interfaces and use these understandings to develop predictive models for macroscopic wetting and flow behavior.

Recent Highlights:

- Molecular Nano-Mechanics - We are employing self-assembled monolayers because they are good models for studying the viscoelastic properties of molecular films and they have demonstrated potential as lubricant layers in micro-machine sensor and actuator devices. Using our acoustic wave damping (AWD) technique, we have made the first ever measurement of the elastic modulus for a single monolayer of di-acetylene molecules: $0.4-1.2 \mathrm{MPa}$ for shear rates of $\sim 0.05-1.0 \mathrm{~m} / \mathrm{sec}$, respectively. At the fastest shear rates, the molecular deformations occur on a time scale that can be accurately modeled by molecular dynamics simulations. Initial results for a perfectly ordered alkane-thiol monolayer confirm our experimental finding of no measurable energy dissipation within the film and predict an alkane chain-length dependence in the elastic modulus. The combination of realistic simulations and AWD experiments will enable us to predict and verify the individual effects of molecular structure, intermolecular bonding, two-dimensional order, and impurity incorporation on the mechanics of the lubricating film. This close coupling between theory and experimental will also provide confidence in this new approach as we extend these measurements to technologically relevant metal and ceramic systems.

- Variable-Temperature Viscoelastic Measurements - We have developed a novel acoustic wave damping technology and a multi-frequency spectrum analysis approach that enables quantitative, temperature-dependent measurements of film or fluid viscoelastic properties with nanometer scale sensitivity. As the sensor temperature is varied over several hundred degrees, resonant frequency shifts due to thermal expansion and discontinuities due to mode exchanges cannot be accurately tracked using conventional oscillator circuits. Our spectrum analysis approach enables us to distinguish these temperature-dependent effects in the sensor from viscoelastic changes in the material under study. This capability is critical for studies of melting, flow, and liquid/solid interfacial reactions.

- Liquid Metal Flow - Real-time video imaging has been used to measure the capillary driven macroscopic flow kinetics of tin/lead solder alloys in well-defined grooves on a copper surface. In contrast to the flow kinetics of alcohols in the same grooves, significant deviations from simple continuum hydrodynamic behavior are observed in the initial stages of liquid metal flow. Fluid momentum, chemical reaction and wall friction effects are being incorporated into new models to account for our results. Molecular dynamics simulations of molten lead using accurate interatomic potentials have been initiated to identify the atomistic processes operative in non-steady state flow and to distinguish physical and chemical contributions in the reactive wetting process.

Future Directions: In the start-up phase of this program we are focused on establishing AWD as a viable technique for measuring the viscoelastic properties of thin layers. This focus places major emphasis on choosing a reproducible and flexible model system (SAM films) and the development of close coupling with theory that can be used interpret AWD results. After we have established this new approach, a complete program will combine AWD viscoelastic measurements, spectroscopic interface characterization, molecular dynamics simulations, and macroscopic flow measurements. Our nanoscale studies will use atomically sharp liquid-metal/solid interfacial structures, followed by chemically reactive and atomically diffuse interfaces, including their temporal evolution. We will use the microscopic results to derive new models to predict complex macroscopic spreading and creep behavior. This approach will be extended to amorphous ceramic films. This systematic progression through increasingly complex but well-defined interfacial structures will enable us to evaluate the role of various energy dissipation mechanisms, interface morphology, impurities, surfactants, and chemical reactions in determining macroscopically observed wetting, spreading, and creep behavior.

Interactions with DOE Technologies: Improving the wetting behavior of liquid metal alloys will greatly increase the reliability of widely used joining technologies such as soldering and brazing. This BES work is providing a scientifically sound understanding of the atomistic flow processes that control solder wetting and flow for high-reliability defense microelectronics applications. This program will establish interactions with DP-funded R\&D and with the Materials Joining Project of the DOE Synthesis and Processing Center.

Program Quality: 1 publication 


\section{Wetting and Flow of Liquid Metals and Amorphous Ceramics at Solid Interfaces KC 020102 \\ Publications}

\section{FY95 Publications}

1. "The Flow of Simple Liquids in Narrow Surface Grooves," J. A. Mann, Jr., L. Romero, R. R. Rye, and F. G. Yost, Physical Review E52, 3967 (1995).

2. "The Flow of Liquids in Surface Grooves," R. R. Rye, J. A. Mann, Jr. and F. G. Yost, Langmuir (in press).

3. "Flow in an Open Channel Capillary," L. Romero and F. G. Yost, J. Fluid Mechanics (submitted). (Joint with NCMS $C R A D A \# 1030 B$ funded by DOE-DP:)

\section{FY96 Publications}

1. "Solder Wetting Kinetics in Narrow V-Grooves," F. G. Yost, R. R. Rye, and J. A. Mann, Jr., Physical Review (submitted). 


\section{Energetic-Particle Synthesis and Science of Materials \\ KC 020103}

Principal Investigators: J.C. Barbour, M.T. Dugger, D.M. Follstaedt, J.A. Knapp, S.M. Myers, C.H. Seager, H.J. Stein and W.R. Wampler

Goals: Use energetic beams and plasmas to create superior new materials and achieve fundamental understanding of the atomic processes, defects, and microstructures that govern materials processing and properties.

Research Highlights:

- Quantification of hard-layer mechanical properties: The intrinsic mechanical properties of submicrometer layers are central to the pursuit of improved tribology. We therefore extended our pioneering use of nanoindentation testing coupled with finite-element modeling, achieving new levels of accuracy and discrimination at high strengths. Our deformation modeling was transferred to the commercial code ABAQUS and extended from two dimensions to more rigorous, three-dimensional analysis. The information content of indentation data was enhanced substantially by detailed tip characterization and the use of rigid substrates. This approach determined yield strengths from 0.04 to $5 \mathrm{GPa}$ and was quantitatively validated by testing pure $\mathrm{Al}$ with known properties.

- New synthesis and understanding of high-strength Al-O nanoalloys: We used pulsed laser deposition (PLD) and electron-cyclotron-resonance (ECR) plasmas in addition to ion implantation to synthesize Al layers containing $0-34$ vol.\% of nanometer $\mathrm{Al}_{2} \mathrm{O}_{3}$ particles and thereby achieved yield strengths up to $5 \mathrm{GPa}$. Newly refined nanoindentation testing coupled with TEM led to a unified understanding of the mechanical response: plasticity through dislocation motion is impeded strongly by the impenetrable oxide particles with a lesser influence of grain boundaries, causing the yield stress of these alloys to vary two orders of magnitude in accordance with theory; the elastic modulus does not depend on dislocation mobility and remains similar to that for pure Al. These insights are widely applicable to nanoalloys.

- Superior new gettering mechanisms for transition metals in $\mathrm{Si}$ : We have now demonstrated that the strongly reactive internal surfaces of voids in $\mathrm{Si}$ are exceptionally effective gettering sinks for $\mathrm{Cu}, \mathrm{Au}, \mathrm{Ni}, \mathrm{Co}$, and $\mathrm{Fe}$ impurities. The binding energies of these metals at cavity walls were quantified, addressing a general lack of information concerning the energetics of transition metals on Si surfaces. Consistent with theoretical considerations, there is a substantial increase in binding energy on going from the multivalent metals to the monovalent adsorbates $\mathrm{Cu}$ and $\mathrm{Au}$. We discovered that precipitates of Si-B phase within Si are even more effective sinks for $\mathrm{Fe}$ than are cavities, with evidence pointing to solutionization of the Fe within the precipitates.

- Location of $\mathrm{H}$ on Si surfaces: Although the technologically important bonding of $\mathrm{H}$ on $\mathrm{Si}$ surfaces has been extensively investigated, the coordinates of the $\mathrm{H}$ atom relative to the $\mathrm{Si}$ lattice were heretofore undetermined. We completed transmission-ion-channeling analyses of chemisorbed $\mathrm{H}$ on both the (100) and (111) Si surfaces to measure these coordinates. Taking the reported relaxation of the Si surface into account, our results indicate a Si-H bond length close to that in the $\mathrm{SiH}_{4}$ molecule.

- BES Center for Synthesis and Processing - Interlaboratory Project on Processing for Surface Hardness: Our advanced capability for materials analysis by high-energy elastic-recoil-detection (ERD) is being used extensively to characterize low-Z refractory layers synthesized in BES programs. Similarly wide exploitation is planned for our newly enhanced finite-element modeling of nanoindentation.

- Relevance to DOE Defense Programs: This program supports DP goals by providing new concepts for reduction of friction and wear in mechanical systems and control of impurities and defects in microelectronics.

\section{Future Directions:}

- Svnthesis of superior new materials using energetic beams and plasmas

- High-strength $\mathrm{Al}$-, Fe-, and Cu-based nanoalloys formed using ion implantation, PLD, and ECR plasmas

- Implanted layers in Si and compound semiconductors for gettering, defect trapping, and electrical isolation

- Low-temperature oxides and nitrides grown from ECR plasmas for surface passivation and optical structures

- Ion-beam studies of fundamental atomic processes, defects. and microstructures

- Trapping mechanisms for detrimental impurities and defects in semiconductors

- Hardening mechanisms and dislocation motion in $\mathrm{Al}, \mathrm{Fe}$, and $\mathrm{Cu}$ nanoalloys

- Injection and bonding of $\mathrm{H}$ in semiconductors as related to passivation of defects, surfaces, and impurities

Program Quality: 12 publications during current reporting period; 4 invited papers in CY 1995 with two at the Int'l Conf. on Ion-Beam Modification of Materials (IBMM'95); IBMM'96 organized by SNL (J.C. Barbour) and LANL (M. Nastasi) 


\section{Energetic-Particle Synthesis and Science of Materials \\ KC 020103}

\section{Publications}

\section{Work Performed under Present BES Program}

1. "Energetic-Particle Synthesis of High-Strength $\mathrm{Al}(\mathrm{O})$ Alloys" (Invited), D.M. Follstaedt, J.C. Barbour, J.A. Knapp and S.M. Myers, Proc. Inter. Conf. on Beam Processing of Advanced Materials (ASM, in press).

2. "Precipitate-Hărdened Aluminum Alloys Formed Using Pulsed Laser Deposition," J.A. Knapp, D.M. Follstaedt and S.M. Myers, J. Appl. Phys. (in press).

3. "Mechanical Properties of High-Strength Aluminum Alloys Formed by Pulsed Laser Deposition," J.A. Knapp and D.M. Follstaedt, Proc. Symp. B, MRS 1995 Fall Meeting (in press).

4. "Low-Energy Deposition of High-Strength $\mathrm{Al}(\mathrm{O})$ Alloys From an ECR Plasma," J.C. Barbour, D.M. Follstaedt, J.A. Knapp, S.M. Myers, D.A. Marshall and R.J. Lad, Proc. Symp. A, MRS 1995 Fall Meeting (in press).

5. "Surface Morphology and Microstructure of Al-O Alloys Grown by ECR Plasma Deposition," D.A. Marshall, J.C. Barbour, D.M. Follstaedt, A.J. Howard and R. J. Lad, MRS 1995 Fall Meeting, Proc. Symp. I (submitted).

6. "Interaction of Copper with Cavities in Silicon," S.M. Myers and D.M. Follstaedt, J. Appl. Phys. (in press).

7. "Diffusion, Precipitation, and Cavity-Wall Reactions of Ion-Implanted Gold in Silicon," S.M. Myers and G.A. Petersen, Proc. Symp. A, MRS 1995 Fall Meeting (in press).

8. "Formation of Cavities in Si and Their Chemisorption of Metals," D.M. Follstaedt and S.M. Myers, Proc. 9th Inter. Conf. on Microscopy of Semiconducting Materials (Inst. of Phys., London, in press).

9. "Cavity Nucleation and Evolution in He-Implanted Si and GaAs," D.M. Follstaedt, S.M. Myers, G.A. Petersen and J.C. Barbour, Proc. Symp. A, MRS 1995 Fall Meeting (in press).

10. "Microstructural Properties of Helium-Implanted Void Layers in Silicon as Related to Front-Side Gettering," J.W. Medernach, T.A. Hill, S.M. Myers and T.J. Headley, J. Electrochem. Soc. (in press).

11. "The Mechanism of Iron Gettering in Boron-Doped Silicon," P.A. Stolk, J.L. Benton, D.J. Eaglesham, D.C. Jacobson, J-Y Cheng, J.M. Poate, S.M. Myers and T.E. Haynes, App. Phys. Lett. (in press).

12. "Oxygen-Related Vibrational Modes Produced in Czochralski Silicon by Hydrogen Plasma Exposure," H.J. Stein and J.W. Medernach, J. Appl. Phys. (in press). 


\title{
Advanced Growth Techniques and the Science of Epitaxy \\ KC 020103
}

\author{
Principal Investigators: E. Chason, J.Y. Tsao, J.A. Floro, B.S. Swartzentruber, E. Heller and S.T. Picraux
}

Goals: To develop advanced growth techniques and in situ diagnostics for fundamental understanding of the science of epitaxy and thin film growth.

Recent Highlights:

- In Situ Film Stress Measurement Technique - (joint with LDRD) Control of stress is critical for the development of advanced thin film device structures, e.g., controlling transport in heteroepitaxial layers and reducing stress-induced voiding in metallization layers. We have developed a laser-based multi-beam technique that enables us to measure wafer curvature and thin film stress in real time during growth. We have obtained an in situ sensitivity of $>5 \mathrm{kM}$ of radius of curvature, corresponding to $<0.01 \%$ strain sensitivity on a standard 2 " Si substrate.

- Measurement of Strain Relaxation during SiGe Heteroepitaxy - The laser curvature technique has been used to measure strain relaxation kinetics in real time during heteroepitaxial growth of SiGe layers on Si. The onset of strain relaxation and subsequent kinetics are being measured to quantitatively test kinetic models of relaxation. Measurements of stress evolution in lattice-mismatched films are enabling us to measure island coalescence during growth.

- Compliant Substrates - (joint with CRADA) The development of stress in thin films can be modified by growing on compliant substrates where lattice mismatch is accommodated by relaxation in the substrate rather than the deposited layers. Our current studies focus on understanding the relaxation mechanism in material grown on SIMOX (Si implanted with oxygen to form a buried amorphous oxide layer) and enhancing relaxation by lowering the oxide viscosity (e.g., via ion implantation). Success will enable the growth of strain-relaxed heteroepitaxial layers without defects for novel micro-electronic (strained Si FET's) and opto-electronic (tuneable band gap) applications.

- In situ Studies of CVD Nucleation and Growth - Although chemical vapor deposition (CVD) is an extremely important growth technique, measurements of growth kinetics have been difficult to obtain with standard surface techniques because of the high ambient gas pressure. We have used X-ray reflectivity to measure real time growth kinetics during deposition of $\mathrm{Fe}$ on $\mathrm{Si}(001)$; results include measurement of 1) transition from nucleation to steady state growth; 2) temperature dependence of nucleation rate; 3) preferential decomposition of precursor on Fe surface compared to Si (autocatalytic effect); 4) onset of island coalescence during growth.

- Formation of Metastable Si(001) Step Structures using Low Energy Ion Bombardment - Using low energy ions, we form a metastable surface comprised of a majority of A-type terraces. The asymmtery between the A and B terrace is determined by a balance between incorporation of defects at the step and elastic step repulsion effects; the asymmetry can be tuned by adjusting the ion flux, temperature and sample miscut.

- UHV-STM on III-V MBE System - We have obtained images of MBE-grown AlAs, GaAs, InAs, AlGaAs and InGaAs using our recently completed UHV STM. The new microscope will be used to study growth kinetics in III-V alloys and the development of lateral composition modulations in strained short-period superlattices.

- DOE Technology Interactions - Understanding and controlling the growth of advanced thin film structures supports DP research and development programs. A patent disclosure has been filed for the laser curvature technique for measuring thin film stress in situ during growth. In our Motorola CRADA, the growth of SiGe layers with controlled strain (using materials input from Sandia) has led to a factor of 12 enhancement in device performance.

Future Directions: Measuring and controlling strain buildup and strain relaxation are a major underlying issue in all thin film growth processes. Using our in situ diagnostics and heteroepitaxial growth capabilities, we will develop techniques using compliant substrates and other novel processes (such as ion-assisted growth) for growing layers with controlled amounts of strain (for example, strain relaxed buffers with low defect densities). We will combine our techniques for measurement of surface structure (X-rays) and film stress (laser curvature) to develop a unique capability for studying the interaction between strain, surface morphology and microstructural evolution. A two dimensional variation of the laser curvature technique will allow stress measurements to be made on III-V wafers during growth. Growth of nitrides on SiC substrates will be studied using a remote plasma source for the nitrogen. The fundamental kinetics of defects (adatoms and surface vacancies) on the Si surface will be measured directly (at temperature and in real-time.) using the new "atom tracker" diagnostic on the STM.

Program Quality: 9 publications, 20 presentations ( 4 invited), 1 patent disclosure. 


\section{Advanced Growth Techniques and the Science of Epitaxy \\ KC 020103}

\section{Publications}

1. Energy Dispersive X-Ray Reflectivity Characterization of Semiconductor Heterostructures and Interfaces, E. Chason, T.M. Mayer, Z. Matutinovic Krstelj and J.C. Sturm, in Semiconductor Characterization, Present Status and Future Needs, W.M. Bullis, D.G. Seiler and A.C. Diebold, eds., (American Institute of Physics, Woodbury, NY, 1996) p. 512.

2. The Role of Transient Ion-Induced Defects in Ion Beam-Assisted Growth, B.K. Kellerman, E. Chason, J.A. Floro, E. Chason, S.T. Picraux and J.M. White, Appl. Phys. Lett. 67, 1703 (1995).

3. Smoothing During Ion-Assisted Growth by Transient Ion Beam-Induced Defects, B.K. Kellerman, E. Chason, J.A. Floro, E. Chason, S.T. Picraux and J.M. White, Mat. Res. Soc. Symp. Proc. Vol. 388, (Pittsburgh, PA, 1995) 349

4. Sputter Roughening Instability on the Ge (001) Surface: Energy and Flux Dependence, E. Chason, B.K. Kellerman and T.M. Mayer, Mat. Res. Soc. Symp. Proc., Fal 1995, submitted.

5. Real-Time Measurement of Epilayer Strain Using a Simplified Wafer Curvature Technique, J.A. Floro, E. Chason and S.R. Lee, Mat. Res. Soc. Symp. Proc., Fal 1995, submitted.

6. Reciprocal Space Analysis of the Initial Stages of Strain Relaxation in Sige Epilayers, S.R. Lee and J.A. Floro, Mat. Res. Soc. Symp. Proc., Fall 1995, submitted.

\section{Joint with other programs}

7. Island Structure Evolution During Chemical Vapor Deposition, D.P. Adams, T.M. Mayer, L.L. Tedder, B.S. Swartzentruber, E. Chason, Surf. Sci., submitted.

8. Direct Measurement of Surface Diffusion Using Atom-Tracking Scanning Tunneling Microscopy, B.S. Swartzentruber, Phys. Rev. Lett, in press.

\section{Ph.D. Student Thesis}

9. Structural Evolution and Surface Reactivity in Semiconductor Processing, B.K. Kellerman, Ph.D. thesis presented to the University of Texas, Austin, TX, 11/95. 


\section{Artificially-Structured Semiconductor Materials Science \\ KC 020105}

Principal Investigators: P. L. Gourley, M. H. Crawford, E. D. Jones, M. B. Sinclair, and J. R. Wendt

Goals: Investigate fundamental structural, optical, and electronic properties of artificially-structured semiconductor materials. These materials include epitaxial layers produced by molecular beam epitaxy or metal-organic vapor phase epitaxy and surface-structured semiconductors produced by photo- or electron beam lithography. The former include quantum wells, superlattices, and distributed Bragg reflectors, one-dimensional photonic lattices, and compositionally modulated structures. The latter include 2-D photonic lattices, and microcavities. The ultimate goals are to identify, synthesize, and study new artificial materials and structures for efficient light generation and collection with emphasis on scientific and technological impact.

\section{Recent Highlights:}

- Lasing on defect states in 1-dimensional photonic lattices: We have studied the threshold for lasing in Bragg heterostructures with an airgap defect for localizing optical modes. Although the pump power for lasing increases by an order of magnitude, airgaps up to 30 microns could support lasing modes. These lasing modes correspond to intense optical fields in a gap between solid surfaces and have important implications for photocatalysis and materials processing or analysis.

- Sandia/NREL Joint Program on Compositional Modulation: Compositionally modified materials have important applications for polarized laser mirror and high-efficiency solar cell design. In order to utilize exiting BES sponsored programs and personal, a joint SNL/NREL program was initiated during FY95-FY96. To date, researchers at SNL have succeeded in producing by MBE, InAs/GaAs structures which exhibit composition modulation. Currently, the growth parameters which lead to optimum modulation depths are being investigated.

- New laser nanoscope for semiconductor studies: By integrating an electron microscope with laser spectroscopy, we have developed methods to simultaneously analyze semiconductor nanostructure and attendant optical properties. Initial studies on photonic lattice nanostructures show that infrared cathodoluminescence is a sensitive tool for assessing effects of ion beam etching in the synthesis of nanostructures, and that visible light spectroscopy/imaging can be performed simultaneously with scanning electron microscopy by secondary electron emission.

- Fabrication of 2-D photonic lattices inside waveguides: Using electron beam lithography and reactive ion beam etching of AlGaAs/GaAs, we have fabricated hexagonal photonic lattices inside optical waveguides. The process uses two separate e-beam pattern-and-etch steps to define the waveguide and then the lattice. During trial fabrication, the e-beam dose, photoresist thickness and hardening, waveguide depth, etch gas pressure and ion beam energy were all systematically varied to optimize the final structure which has near perfect cylindrical, $200 \mathrm{~nm}$ posts on $400 \mathrm{~nm}$ centers.

- Phonon side-bands in Quantum well magnetoluminescence: We have developed a theory to describe the first experimental observation of phonon replicas for optical transitions between electron and hole Landau levels in quantum wells. The theory is in excellent agreement with magneto luminescence spectra that we have recorded for InGaAs/GaAs strained-layer quantum wells. These spectroscopic analyses serve as powerful tools in the characterization of semiconductor materials.

- Ultrafast transport measurements of carriers in visible, light-emitting quantum wells: Using sequences of quantum wells of different bandgaps, we can artificially impede the energy relaxation of electrons and holes to the ground state of a semiconductor. Ultrafast photoinduced absorption has been used to obtain temporal waveforms of bleaching transients of these states. These data indicate that the ground state rise time is consistent with the time required for diffusion from the surface where they were created. Thus good mobility, essential for efficient light emission, can be achieved with inhibited relaxation.

- Advanced Photovoltaics for the Center for Synthesis and Processing: Control of hot-electron relaxation could lead to a very low threshold laser or LED, and also very high efficiency $(-60 \%)$ solar cells. A joint SNL/NREL program to investigate hot electron relaxation mechanisms under the High Efficiency Photovoltaic Project of the BES S\&P Center has been initiated.

Future Directions: Near infrared and visible bandgap alloys and quantum well heterostructures will continue to be developed by metal-organic vapor phase epitaxy and molecular beam epitaxy. We will develop new spectroscopic and microscopic methods for assessing optical transitions and energy states, carrier mobility, and structural quality of epitaxial layers. New methods for fabricating semiconductor microstructures and nanostructures for near IR and visible materials will be investigated.

Program Quality: Invited talks, 3 invited review articles including Scientific American article, DOE Nanophotonics Workshop hosted by Sandia August, 1995, 2 patent applications, 12 publications, personnel selected for Board of Advisors to Micro-optical Devices, Inc, committee member International Quantum Electronics Conference, international collaboration through NATO Advanced Study Institute, organized MRS Symposium on Ordering and Composition Modulation in Optoelectronic Materials, Fall, 1995. 


\section{Artificially Structured Semiconductor Materials Science \\ KC 020105}

\section{Publications}

1. Semiconductor Microstructures for Efficient Light Generation, P. L. Gourley, Scientific American, invited review, in press.

2. Intracavity Dielectric Spectroscopy with Vertical Cavity Surface-emitting Lasers, K. E. Meissner, P. L. Gourley, T. M. Brennan, B. E. Hammons, and A. E. McDonald, Applied Physics Letters, submitted.

3. Intracavity Spectroscopy of Dielectric Spheres in Semiconductor Microcavities, K. E. Meissner, P. L. Gourley, T. M. Brennan, B. E. Hammons, and A. E. McDonald, Applied Physics Letters, Proceedings of SPIE Photonics West '95, Microcavity Semiconductor Structures, San Jose, 1995.

4. Effects of Quantum Well Subband Structure on the Temperature Stability of Vertical Cavity Semiconductor Lasers, W. W. Chow, K. D. Choquette, and P. L. Gourley, Applied Physics Letters 663266 (1195).

5. Transient Photo-induced Absorption in AlGaAsP Fractal Quantum Well Heterostructures, M. B. Sinclair, P. L. Gourley, M. H. Crawford, K. E. Meissner, and R. P. Schneider, Jr. Applied Physics Letters, submitted.

6. Near Infrared, Subwavelength Diffractive Nanostructures Fabricated with Semiconductors, J. R. Wendt, R. E. Smith, M. E. Warren, G. A. Vawter, Proceedings of SPIE Photonics West'95, San Jose, 1995.

7. Magneto-Optical Studies of Compound Semiconductors, E. D. Jones, J. F. Klem, and S. K. Lyo, J. Phys. and Chem. Solids 56 (1995).

8. Suppression of Direct-transition Phonon Side Bands in the Magnetoluminescence from Doped Quantum Wells, S. K. Lyo, E. D. Jones, and J. F. Klem, Phys. Rev. Lett., submitted.

9. Determination of Band Structure Dispersion Curves by Optical Techniques, E. D. Jones, S. K. Lyo, and J. F. Klem, Proc. 22nd International Symposium on Compound Semiconductors, in press (1995).

10. Compositional Dependence of luminescence of InAlGaP alloys near the direct-indirect bandgap cross-over, J. S. Nelson, E. D. Jones, S. M. Myers, D. M. Follstaedt, R. P. Schneider, Jr., J. A Schirber, H. P. Hjalmarson, J. Fouquet, V. Robbins, K. Carey, J. Appl. Phys. (1995). 


\section{Physics and Chemistry of Novel Superconductors \\ KC 020202}

Principal Investigators: T. L. Aselage, N. A. Missert, B. Morosin, P. P. Newcomer, J. E. Schirber, M. P. Siegal, E. L. Venturini

Goals: Develop a fundamental understanding of the physics and chemistry of novel superconductors with emphasis on $\mathrm{TIBaCaCuO}$ materials; synthesize the highest quality thin films and single crystals for basic research; investigate intrinsic superconducting properties, particularly the complex relationship between transport, magnetic behavior and microstructure.

Research Highlights:

- High-temperature phase equilibria: High-resolution transmission electron microscopy (HRTEM) of two-zone equilibrated Tl-1223 has identified Tl-1234 as the prevalent intergrowth structure. From this information, two-zone conditions have been refined to nearly eliminate the intergrowths, thereby establishing the equilibrium boundaries on $\mathrm{Tl}_{2} \mathrm{O}$ and $\mathrm{O}_{2}$ partial pressures for synthesizing the Tl-1223 phase and determining its superconducting properties.

- TIBaCaCuO film microstructure and properties: Furnace annealing of Tl-1212 films at 600EC in nitrogen produces a substantial increase in $\mathrm{J}_{\mathrm{c}}$ and magnetic vortex pinning. HRTEM has identified $n \mathrm{~nm}$-sized defects introduced by the anneal with a density and orientation that could explain the enhanced pinning.

- Irreversibility crossover for Tl-2212 film and crystal: The irreversibility lines for a Tl-2212 film $\left(\mathrm{T}_{\varepsilon} \sim 101 \mathrm{~K}\right)$ and single crystal $\left(T_{c} \sim 110 \mathrm{~K}\right)$ exhibit a crossover near 1 tesla and $T / T_{c}=0.5$. The vortex lattice in the film is stable to higher temperatures at low fields but not at fields above 1 tesla. This explains the higher self-field $\mathrm{J}_{\mathrm{c}}$ in the film at $77 \mathrm{~K}$.

- IlBaCaCuO film synthesis: We scaled-up our two-zone process to grow uniform $\mathrm{Tl}$ films on large-area substrates to enable studies of microwave surface resistance and power handling.

- Single crystal irradiation: High-energy ion-induced microstructural modification of Tl-1212 and Tl-2212 crystals was studied in situ by electron diffraction and post irradiation by HRTEM. The fluence required to amorphize or completely alter the structure rises both at high temperature and below $T_{c}$. The latter unexpected result is attributed to the reduced thermal conductivity in the superconducting state.

- New Materials: (a) We have demonstrated that for $\mathrm{Na}_{2} \mathrm{CsC}_{60}$ certain pressure/temperature protocols result in the reversible formation of a superconducting "polymeric" orthorhombic structural phase, challenging earlier theoretical models for such alkali-intercalated $\mathrm{C}_{60}$ compounds. (b) An alternative synthesis route for the oxyfluoride superconductors $\left(\mathrm{Sr}_{1-x} \mathrm{M}_{x}\right)_{2} \mathrm{CuO}_{2} \mathrm{~F}_{2+d}[\mathrm{M}=\mathrm{Ba}, \mathrm{Ca}]$ was explored. $\mathrm{ES} \& \mathrm{H}$ concerns posed by direct $\mathrm{F}_{2}$ synthesis were mitigated by using either $\mathrm{NH}_{4} \mathrm{~F}$ or $\mathrm{NH}_{4} \mathrm{HF}_{2}$ with $\left(\mathrm{Sr}_{1-\mathrm{x}} \mathrm{M}_{\mathrm{x}}\right)_{2} \mathrm{CuO}_{3}$ precursors in sealed quartz tubes. These materials have apical fluoride ions which could help elucidate the role of the apical ions in high-temperature superconductivity. (c) The crystal structure of the recently discovered heavy-fermion compound, $\mathrm{Ce}_{2} \mathrm{Pt}_{6} \mathrm{Ga}_{15}$, was determined from combined neutron and $\mathrm{x}$-ray diffraction data. The complex cubic close-packed structure is unusual: one-third of the $\mathrm{Ce}$ atoms in the $\mathrm{Ce}$ layers are replaced by groups of three $\mathrm{Ga}$ atoms in an ordered manner. This explains the observed poor resistance ratio and provides a structural basis for the extremely interesting, but poorly understood, magnetic and transport properties.

Future Directions: We will continue our integrated study of phase dynamics, synthesis and the physics of various HTS materials, emphasizing the $\mathrm{TlBaCaCuO}$ system. Our goals are to understand the intrinsic properties of single crystals and to optimize the electronic properties of epitaxial thin films for device applications through control of the microstructure. Synthesis and characterization of other cuprates and novel compounds will be pursued to provide an experimental basis for validating proposed theories of intrinsic properties of high-temperature superconducting materials.

\section{Technical Interactions:}

Defense Programs: superconducting energy storage and switching for enablement in surety component development; portable voltage standard. Energy Efficiency and Renewable Energy: applying phase equilibria and two-zone processing expertise to Tl-based thick films and tapes. Active research collaborations with universities: Maryland, New Mexico, Pennsylvania and Wisconsin; industry: AT\&T Bell Labs and DuPont CR\&D; government labs: synthesis, phase separation and microstructure (LANL, Ames), neutron scattering (ANL, LANL), intergrowths and microstructure (ANL), vortex motion in crystals (ANL), Josephson devices (NIST) and photoemission on films (JPL).

Tech Transfer: Research supported by this BES program prompted and continues to leverage a Technology Transfer Initiative CRADA with DuPont to develop a new manufacturing process for $\mathrm{Tl}$-based high- $\mathrm{T}_{\mathrm{c}}$ superconducting thin films.

Program Quality and Impact: 1 patent disclosure, 5 invited presentations, 16 publications. 


\title{
Physics and Chemistry of Novel Superconductors \\ KC 020202
}

\author{
Publications
}

\section{Under BES support at Sandia:}

1. Growth of $\mathrm{TlBa}_{2} \mathrm{CaCu}_{2} \mathrm{O}_{7}$ thin films using a controlled Tl-oxide source during processing, M. P. Siegal, N. Missert, E. L. Venturini, P. P. Newcomer, F. Dominguez, R. Dunn, IEEE Trans. Appl. Supercond. 51343 (1995).

2. Importance of controlling the Tl-oxide partial pressure throughout the processing of $\mathrm{TlBa}_{2} \mathrm{CaCu}_{2} \mathrm{O}_{7}$ thin films, $\mathrm{M}$. P. Siegal, E. L. Venturini, P. P. Newcomer, D. L. Overmyer, F. Dominguez, R. Dunn, J. Appl. Phys. 787186 (1995).

3. Enhancement of the superconducting properties of $\mathrm{TlBa}_{2} \mathrm{CaCu}_{2} \mathrm{O}_{7^{+}}$thin films via post-annealing, M. P. Siegal, E. L. Venturini, P. P. Newcomer, B. Morosin, D. L. Overmyer, F. Dominguez, R. Dunn, Appl. Phys. Lett. 673966 (1995).

4. Ion beam modification of $\mathrm{TlBaCaCuO}$ high-temperature superconductors during irradiation, P. P. Newcomer, B. Morosin, Mater. Res. Soc. Proc. 373425 (1995).

5. Stability of the Tl-1223 phases, T. L. Aselage, E. L. Venturini, J. A. Voigt and D. J. Miller, J. Mater. Res., in press.

6. Temperature-dependent microstructural modification in ion-irradiated Tl-type high-temperature superconductors, P. P. Newcomer, L.-M. Wang, J. C. Barbour, R. C. Ewing, M. L. Miller, B. Morosin, submitted to Physica C.

7. Effect of pressure on the superconducting primitive cubic and "polymeric" phases of $\mathrm{Na}_{2} \mathrm{CsC}_{60}, \mathrm{~J}$. E. Schirber, L. V. Hansen, B. Morosin, J. E. Fischer, J. D. Jorgensen, G. H. Kwei, submitted to Physica C.

\section{Collaborative - Sandia work supported by BES:}

8. Correlation of vortex motion in high- $T_{c}$ superconductors, T. S. Lee, N. Missert, L. T. Sagdahl, J. Clarke, J. R. Clem, K. Char, J. N. Eckstein, D. K. Fork, L. Lombardo, A. Kapitulnik, L. F. Schneemeyer, J. V. Waszczak, R. B. van Dover, Phys. Rev. Lett. 742796 (1995).

9. Temperature dependence and magnetic field modulation of critical currents in step-edge SNS YBCO/Au junctions, $\mathrm{N}$. Missert, L. R. Vale, R. H. Ono, C. D. Reintsema, D. A. Rudman, R. E. Thomson, S. J, Berkowitz, IEEE Trans. Appl. Supercond. $\underline{5} 2969$ (1995).

10. The critical current and normal resistance of high- $T_{c}$ step-edge SNS junctions, C. D. Reintsema, R. H.Ono, G. Barnes, L. Borcherdt, T. E. Harvey, G. Kunkel, D. A. Rudman, L. R. Vale, N. Missert, P. A. Rosenthal, IEEE Trans. Appl. Supercond. $\underline{5} 3405$ (1995).

11. Oxygen ordering and phase separation in $\mathrm{La}_{2} \mathrm{CuO}_{4+x}$, B. W. Statt, P. C. Hammel, Z. Fisk, S.-W. Cheong, F. C. Chou, D. C. Johnston, J. E. Schirber, Phys. Rev. B $\underline{52} 15575$ (1995).

12. Aspects of macroscopic phase separation and interstitial oxygen ordering in oxygen-doped $\mathrm{La}_{2} \mathrm{CuO}_{4+\mathrm{x}}, \mathrm{P}$. C. Hammel, B. W. Statt, F. C. Chou, D. C. Johnston, Z. Fisk, S.-W. Cheong, J. E. Schirber, Conf. on Anharmonic Properties of High $T_{c}$ Cuprates (1995), in press.

13. Structure of $\mathrm{Ce}_{2} \mathrm{Pt}_{6} \mathrm{Ga}_{15}$; interplanar disorder from $\mathrm{Ce}_{2} \mathrm{Ga}_{3}$ layers, G. H. Kwei, A. C. Lawson, A. C. Larson, B. Morosin, E. M. Larson, P. C. Canfield, submitted to Acta Crystall.

14. Synchrotron $x$-ray powder diffraction study of electrochemically oxidized $\mathrm{La}_{2} \mathrm{CuO}_{4+x}$ and $\mathrm{La}_{2-\mathrm{x}} \mathrm{Nd}_{\mathrm{x}} \mathrm{CuO}_{4+\mathrm{x}}, \mathrm{M}$. K . Crawford, R. L. Harlow, E. M. McCarron, N. Herron, W. E. Farneth, W. J. Donahue, B. A. Parkinson, J. E. Schirber, J. Phys. Chem. Solids, in press.

15. Synthesis and characterization of two new organic superconductors: $6_{\mathrm{L}}-$ and $6_{\mathrm{H}}-(\mathrm{ET})_{2} \mathrm{Au}\left(\mathrm{CF}_{3}\right)_{4}(1,2,2$-trichloroethane $)$ via microcrystallization [ET = bis(ethylenedithio)tetrathiafulvalene], J. A. Schlueter, J. M. Williams, U. Geiser, J. D. Dudek, S. Shirchio, M. Kelly, J. Gregar, W. Kwok, J. Fendrich, J. E. Schirber, W. R. Bayless, D. Naumann, T. Roy, J. Chem. Soc. Chem. Commun., in press.

16. New ET-based organic superconductors with the organometallic anion $\mathrm{Cu}(\mathrm{CF} 3) 4, \mathrm{U}$. Geiser, J. A. Schlueter, K. D. Carlson, J. M. Williams, H. H. Wang, W.-K. Kwok, U. Welp, J. Fendrich, J. Dudele, C. Achenbach, A. Komora, P. Keone, D. Naumann, T. Roy, J. E. Schirber, W. R. Bayless, J. Ren, M.-H. Whangbo, Intern. Conf. Sci. Techn. of Synthetic Metals, to appear in Synth. Metals. 


\title{
Tailored Surfaces and Interfaces for Materials Applications \\ KC 020202
}

\author{
Principal Investigators: G. L. Kellogg, P. J. Feibelman, T. M. Mayer, B. S. Swartzentruber, N. D. Shinn
}

Goals: To understand the atomic-level processes that control the growth and stability of materials surfaces and to use this understanding to tailor the structural and electronic properties of surfaces and interfaces for advanced materials applications.

\section{Recent Highlights:}

- Ballistic Atom Exchange during Low-Temperature Deposition - A non-thermal or "ballistic" exchange process between vapor-deposited atoms and substrate surface atoms has been observed by field ion microscopy. A fraction (11\%) of Ir atoms impinging on a $\mathrm{Rh}(100)$ single-crystal surface embed themselves into the top layer of surface atoms during condensation at $77 \mathrm{~K}$. Observation of an exchange process at temperatures well below those required for thermal exchange $(330 \mathrm{~K})$ provides compelling evidence that an atom can transfer some of its energy of condensation into motion along the surface. The existence of this phenomenon (transient mobility) has been a subject of considerable controversy in recent years.

- Direct Measurements of Si Surface Diffusion on Si(001) - Using Sandia's newly developed atom-tracker STM, we have measured the temperature-dependent diffusion rate of Si dimers on the Si(001) surface. We show that mass-transport kinetic pathways are quite complicated involving highly anisotropic cluster migration and atom-defect interactions that dominate the long-range diffusion process. These fundamental understandings are necessary to develop and validate realistic simulations and models of the growth of semiconductor materials.

- Energetics of Steps on Pt(111) - We have determined the step energies per unit length for the two types of unkinked steps on the $\operatorname{Pt}(111)$ plane from first-principles LDA optimizations of $\operatorname{Pt}(331), \operatorname{Pt}(221) \operatorname{Pt}(211)$, and $\operatorname{Pt}(533)$ slabs. The calculated energies of both the (111)- and (100)-type steps are nearly equal, about $0.46-0.47 \mathrm{eV}$ per step-edge atom. Quantitative determinations of step energies are important in that these energies define the equilibrium shape of islands during thin-film growth.

- Hydrogen-Enhanced Atom Mobility on Surfaces - First-principles calculations of the interaction of hydrogen with the $\mathrm{Be}(0001)$ surface reveal that adsorbed $\mathrm{H}$ reduces the surface diffusion barrier for $\mathrm{Be}$ on $\mathrm{Be}(0001)$ by a factor of three. The adsorbed $\mathrm{H}$ weakens the bonds of the atom to the surface and acts like a "skyhook" to lift it away from the surface. These calculations provide new insight into the mechanisms by which adsorbates change the kinetics and/or energetics of materials growth.

- Surface Passivation for Selective Area Growth - We have been able to alter surface reactivity and nucleation kinetics in chemical vapor deposition of metals on $\mathrm{Si}(001)$ by passivating reactive sites with adsorbed hydrogen. Si dangling bond sites terminated with $\mathrm{H}$ exhibit much higher activation energy for decomposition of $\mathrm{Fe}(\mathrm{CO}) 5$ precursors than free dangling bond sites. We have further demonstrated that nanometer-scale areas on passivated surfaces where the passivating $\mathrm{H}$ has been removed by electron-induced desorption serve as selective nucleation sites for Fe growth. Adsorbed $\mathrm{H}$ can be used as an atomic layer resist for nanostructure fabrication using this process.

Future Directions: This program will continue to focus on understanding the microscopic mechanisms that control the growth of thin surface films. Atomic-scale experimental and theoretical investigations will provide detailed information on the energetics of elementary steps in the growth process and determine how ideal growth behavior is modified by the presence of surface defects and chemical adsorbates (surfactants). Comprehensive microscopic models of morphology development in CVD of metals on Si will be lead to nanometer-scale selective area CVD growth by chemical manipulation of nucleation processes. Sandia's newly developed "atom-tracker" STM will enhance our experimental efforts by allowing us to use an individual atom as a probe of surface interactions. New capabilities in Low Energy Electron Microscopy (LEEM) will also be added to the program to enhance our real-time analysis of dynamical surface processes and to test predictions based on our atomic-level understanding.

Interactions with DOE Technologies: This BES-supported research is providing both fundamental understanding and quantitative input required to develop science-based models of materials growth and stability. Interactions with DP-funded R\&D programs addressing the long-term stability of materials will lead to more reliable predictions of component reliability and safety.

Program Quality: 14 Publications, 8 Invited Presentations at National and International Conferences, 10 Invited Presentations at Universities/Research Labs 
Tailored Surfaces and Interfaces for Materials Applications

KC 020202

\section{Publications}

\section{Work Performed under BES Program}

1. "Experimental Observation of Ballistic Atom Exchange on Metal Surfaces," G. L. Kellogg, Phys. Rev. Lett. 76,98 (1996).

2. "Field Ion Microscope Investigations of Adatom and Dimer Diffusion along Rh(100) Step Edges," G. L. Kellogg, Surface Science (in press).

3. "Atomic-Level Studies of Cluster Diffusion on Metal Surfaces," G. L. Kellogg, Proc. of Int. Symposium on the Science and Technology of Atomically Engineered Materials, P. Jena, editor, (World Scientific, Singapore/London, 1996) (in press).

4. "Scanning Tunneling Microscopy - Energetics from Statistical Analysis," Peter J. Feibelman, Phys. Rev. B 5212444 (1995).

5. "Energetics of Steps on Pt(111)," Peter J. Feibelman, Phys. Rev. B (in press).

6. "Relaxation of hcp(0001) Surfaces: A Chemical View," Peter J. Feibelman, Phys. Rev. B (in press).

7. "Disagreement between Experimental and Theoretical Metal Surface Relaxations," Peter J. Feibelman, Surface Science (in press).

8. "Book Review: Concepts in Surface Physics, by M. C, Desjonqueres and D. Spanjaard," Peter J. Feibelman, Adv. Mat. I, 328 (1995).

9. "Selective Area Growth of Metal Nanostructures," D. P. Adams, T. M. Mayer, B. S. Swartzentruber, Appl. Phys. Lett. (submitted).

10. "Island Structure Evolution During CVD Growth", D. P. Adams, T. M. Mayer, E. Chason, B. K. Kellerman, B. S. Swartzentruber, Surf. Sci. (submitted).

11. "Decomposition of $\mathrm{P}\left(\mathrm{CH}_{3}\right)_{3}$ on $\mathrm{Ru}(0001)$ : Comparison with $\mathrm{PH}_{3}$ and $\mathrm{PCl}_{3}$," H.-S. Tao, U Diebold, N. D. Shinn and T. E. Madey, Surface Science (submitted).

Performed jointly with other programs

12. "Direct Measurement of Surface Diffusion Using Atom-Tracking Scanning Tunneling Microscopy," B. S. Swartzentruber, Phys. Rev. Lett. (in press).

13. "H-enhanced Mobility and Defect Formation at Surfaces: $\mathrm{H}$ on Be(0001)," Roland Stumpf, Phys. Rev. B (in press).

14. "Nanometer-Scale Lithography on $\mathrm{Si}(001)$ using Adsorbed $\mathrm{H}$ as an Atomic Layer Resist", D. P. Adams, T. M. Mayer, B. S. Swartzentruber, J. Vac. Sci. Technol. B (submitted). 
Goals: To understand and exploit novel aspects of the electronic transport of complex molecular, network and multicomponent materials arising from their unusual structures and bondings.

Highlights:

- Boron carbides Hall mobilities are found to be low and thermally assisted showing transport to be by hopping.

- Seebeck coefficients of boron carbides were measured from $15 \mathrm{~K}$ to $900 \mathrm{~K}$. The Seebeck coefficients rise with increasing temperature to a plateau $(>180 \mu \mathrm{V} / \mathrm{K})$. These high-temperature Seebeck coefficients are very much larger than those of conventional materials with comparable carrier densities. The temperature dependences of these Seebeck coefficients are also unusual. Large Seebeck coefficients with such temperature-dependences are shown consistent with hopping-type carriers softening the twelve-atom icosahedra between which they hop.

- The magnetic-field dependence of the low-temperature Seebeck coefficient measures a carrier's magnetic moment. The absence of a magnetic-field dependence of boron carbides' low-temperature Seebeck coefficients in our measruements at a field of $10 \mathrm{~T}$ provides direct evidence of the carriers being singlet pairs.

- Carrier densities in boron carbides far exceed densities of (defect-related) localized spins observed in low-temperature susceptiblity measurements. Thus, the carriers pair as singlets. We have extended ESR measurements to $1000 \mathrm{~K}$ to look for the breaking of small-bipolarons' singlet pairs. Above room temperature we observed a thermally generated spin density having a $g$ value that differs from that of the defect-related spins.

- Our theory of high-temperature small-bipolaron hopping shows that singlet bipolarons primarily break and hop separately as small polarons before recombining. This pair-breaking affects the conductivities, Seebeck coefficients and magnetic susceptibilities in ways consistent with our observations of boron carbides. We therefore associate thermally generated "new" spins observed in ESR with breaking of singlet bipolarons.

- We measured the Raman spectra of isotope-enriched $\mathrm{B}_{12} \mathrm{O}_{2}$. Since we observed no shifts of the features assigned to $\mathrm{B}_{12}$ motions with oxygen isotope, "icosahedral" modes of icosahedral borides' Raman spectra are not affected by motion of non-icosahedral (oxygen) atoms. Thus, the shifts of the icosahedral modes of boron carbides with carbon isotope imply carbon atoms substituting for boron atoms within icosahedra $\left(\mathrm{B}_{11} \mathrm{C}\right.$ icosahedra).

- We studied the effect of electron correlation on the electronic charge distribution of a large bipolaron confined to a plane embedded within a strongly ionic medium. For wide electronic bands the Coulomb repulsion between the bipolaron's two carriers drives them into mutually perpendicular dumbbell-shaped charge distributions. The four-lobed shapes of these bipolarons should reflect itself in the ordering parameters of their condensate.

\section{Future Directions:}

- Experimental and theoretical efforts on borides will be expanded to include unusual transport of other complex solids: clathrate networks and compositionally complex pyrochlores. Nonohmic hopping-type conduction in disordered systems will be calculated without the resistor-network approximation since it fails at large electric fields and low temperatures. Low-temperature/high-field measurements of boron carbides' hopping conduction will begin. -The non-Arrhenius hopping transport we measure in boron carbides below room-temperature will be analysed in terms of 1) freezing-out of the pair-breaking mode of small-bipolaron hopping, 2) freezing out of multiphonon hopping, and 3) percolation effects associated with boron carbides' disorder. - Electrical measurements of boron carbides will be extended up to $1800 \mathrm{~K}$. $\cdot$ We will try to test our model of carbon concentrations of boron carbides' icosahedra with ${ }^{13} \mathrm{C} N \mathrm{NMR}$. $\bullet \mathrm{AC}$ conductivity measurements will test whether $\beta$-boron's surprisingly low dc conductivity results from most carriers being bound in isolated elements of $\beta$-boron's complex structure. - The high-temperature ac conductivity of carriers in deep traps will be calculated.

Relations to DOE Technologies: Understanding boron-rich solids supports their use in neutron absorbers and detectors, thermoelectric energy conversion, radiation-hard semiconductors, and first-wall MFE materials. Recently supersaturated boron densities implanted in silicon, when heated, are found to form insulating boron-rich precipitates that getter metal impurities, thereby suggesting an in situ means of "purifying" silicon semiconductors.

Program Quality: Invited to: write reviews for Rev. of Mod. Phys. and for Intl. J. of Mod. Phys., and edit book on Boronrich Solids; Members of committees on Boron, Borides \& Related Compounds, Boron in USA, Hopping \& Related Phenomena; 5 invited talks, 13 publications 


\section{Transport in Unconventional Solids \\ KC 020205}

\section{Publications}

\section{PUBLICATIONS}

1. Boron Carbides, T. L. Aselage and D. Emin, in CRC Handbook of Thermoelectrics, D. M. Rowe ed. (CRC Press, Boca Raton, 1995) pp. 373-387.

2. Defect Clustering and Self-Healing of Electron-Irradiated Boron-Rich Solids, M. Carrard, D. Emin and L Zuppiroli, Physical Review B 51, 11,270 (1995).

3. Neutron Powder Diffraction Refinement of Boron Carbides: Nature of Intericosahedral Chains, B. Morosin, G.H Kwei, A.C. Lawson, T.L. Aselage and D. Emin, Journal of Alloys and Compounds $\underline{226}, 121$ (1995).

4. Observation of Inverted-Molecular Compression in Boron Carbide, R. J. Nelmes, J. S. Loveday, R. M. Wilson, W. G. Marshall, J. M. Besson, S. Klotz, G. Hamel, T. L. Aselage and S. Hull, Physical Review Letters 74, 2268 (1995)

5. Synthesis and Cell Refinement for Icosahedral Boron Phosphide $B_{12} P_{2}, P$. Yang and T. L. Aselage, Powder Diffraction 10, 263 (1995).

6. Small-Bipolarons in Boron Carbides: Pair Breaking in Semiclassical Hopping, David Emin in Hopping and Related Phenomena, O. Millo and Z. Ovadyahu editors (Raccah Institute of Physics, The Hebrew University, 1995) pp. 131136.

7. Formation, Phase Separation and Superconductivity of Large Bipolarons, David Emin in Polarons and Bipolarons in High- $\mathrm{T}_{\underline{c}}$ Superconductors and Related Materials, E. S. H. Salje, A. S. Alexandrov and W.Y. Liang, eds., (Cambridge University Press, 1995) pp. 80-109.

8. Large Bipolarons and Superconductivity, David Emin, Journal of Superconductivity, $\underline{8}, 523$ (1995).

9. Effect of Electronic Correlation on the Shape of a Large Bipolaron: Four-Lobed Planar Large Bipolaron in an Ionic Medium, David Emin, Physical Review B 52, 13874 (1995).

10. Large Bipolarons and Superconductivity, David Emin in Molecular Physics Reports (in press).

11. Pair Breaking in Semiclassical Singlet Small-Bipolaron Hopping, David Emin, Physical Review B (in press, scheduled January 15,1996$)$.

12. Spin Susceptibility of Boron Carbides: Dissociation of Singlet Small-Bipolarons, O. Chauvet, D. Emin, L. Forro, L. Zuppiroli and T. L. Aselage, Physical Review B (submitted).

13. Raman Spectra of Isotopically Enriched $B_{12} A_{2}, B_{12} P_{2}, B_{12} O_{2} A N D B_{12+x} C_{3-x}$ : Compositions and Relative Stiffness of Icosahedra and Chains, T. L. Aselage, D. R. Tallant and D. Emin, Physical Review B, (submitted). 


\section{CVD Sciences}

KC 020303

Principal Investigators: P. Esherick, M. E. Bartram, W. G. Breiland, M. E. Coltrin, J. R. Creighton, P. Ho, K. P. Killeen, H. K. Moffat, R. J. Kee, F. Rupley

Goals: To understand the basic physics and chemistry of thin-film synthesis by chemical vapor deposition (CVD). This includes the development of new measurement techniques and theoretical tools useful for both fundamental scientific studies and for improvements of CVD processes and equipment.

\section{Recent Highlights:}

- Chemistry of GaAs and AlAs growth by MOCVD: A group V inhibition of the surface reaction rate during the MOCVD of GaAs and AlAs from TMGa, TMAl, $\mathrm{AsH}_{3}$, and $\mathrm{tBuAs}$ precursors has been experimentally observed in a rotating disk reactor. We successfully modeled the growth rate data with a kinetics mechanism involving the partial formation of a $c(4 \times 4)$ layer of As on the surface which blocks incorporation of the group III species onto its normal surface adsorption site.

- Site-Independent TEOS Reactions on $\mathrm{SiO}_{2}$ at CVD Temperatures and Pressures: We have shown with FTIR and isotopic labeling that TEOS decomposition rates are independent of surface site coverages on $\mathrm{SiO}_{2}$ at $1000 \mathrm{~K}$. This lack of sitespecificity suggests that a precise knowledge of the coverages of siloxane bridges and silanols on $\mathrm{SiO}_{2}$ is not required for modeling surface reaction rates in TEOS-based CVD processes.

- Program Initiated in MOCVD of Group-III Nitrides Films: We began research on the metal-organic CVD growth of Group-III-nitride films (e.g. GaN, AIN, InGaN, etc.) in a rotating disk reactor. Growth rate measurements were made as a function of reactant partial pressures and substrate temperature using in situ spectral reflectance as a real-time monitor. The evolution of surface roughness throughout film growth was observed using a combination of reflectance and light scattering probes.

- In situ growth rate monitors: The ADVISOR (Analysis of Deposition with Virtual Interfaces and Spectroscopic Optical Reflectance) method was generalized to include rough film surfaces. This allows us to explore surface nucleation, evolution of morphology, and important compound semiconductor film properties such as lattice matching and relaxation. The ADVISOR method has also been re-cast in a formalism that uses Bayes or Kalman filters to extract film thickness and composition in real time.

- Enhanced modeling capabilities for simulation of surface chemistry: We have significantly enhanced the modeling capabilities of our Surface Chemkin software to facilitate modeling of plasma/surface interactions. Previous versions of the software based chemical reaction rates on a thermodynamic temperature, which may not be defined in plasma systems. We have modified the formalism to allow the rate of reaction to depend upon collision energies of individual species. This change required many structural changes in the code, the most significant of which is allowing for a variation in "temperature" between different species.

- Spectroscopic identification of methyl binding sites on GaAs: We have shown that we can differentiate methyl $\left(\mathrm{CH}_{3}\right)$ groups bonded to $\mathrm{Ga}, \mathrm{Al}$, and As using the symmetric $\mathrm{C}-\mathrm{H}$ bending mode $\left(\delta_{\mathrm{s}}\right)$ as the fingerprint. Although the $\mathrm{C}-\mathrm{H}$ stretching modes of all species are similar, the $\mathrm{C}-\mathrm{H}$ bending modes for surface $\mathrm{Ga}-\mathrm{CH}_{3}, \mathrm{Al}-\mathrm{CH}_{3}$, and As- $\mathrm{CH}_{3}$ are measured (with surface FTIR) to be 1192,1214 , and $1251 \mathrm{~cm}^{-1}$, respectively. Using this knowledge the transfer of $\mathrm{CH}_{3}$ ligands from organometallic adsorbate to surface atoms was directly observed.

- Reflectance-difference spectroscopy (RDS) installed on surface science system: We have measured the RD spectra of well characterized $\mathrm{CH}_{3}$ and $\mathrm{CH}_{2}$ terminated $\mathrm{GaAs}$ surfaces. We will compare these results to measurements obtained under true MOCVD conditions to test for the presence of these adsorbates. We also determined that a new and uncharacterized RDS lineshape seen for some MOCVD conditions (by Aspnes and others) corresponds to a slightly altered form of the c(4X4) "super" arsenic-rich surface.

Future Directions: As a new direction we are initiating a research effort in wide-bandgap group-III nitrides (e.g. InGaAIN) for high-temperature electronics and efficient visible/UV light sources. We will continue to pursue development of new diagnostic tools and modeling capabilities to probe the details of deposition chemistry relevant to CVD, with our emphasis on in situ techniques to study molecular species reacting at surfaces. As an extension of our chemistry modeling activities, we will extend the CHEMKIN formalism to address reactions within the solid state including the oxidation of semiconductors and metals. Such reactions have broad significance ranging from corrosion processes to microelectronic fabrication to the synthesis of high-Tc superconductors and photovoltaics.

Program Quality: 15 publications, 29 presentations, including 12 invited talks at national or international symposia. 


\section{CVD Sciences}

KC 020303

\section{Publications}

\section{Work performed under present BES Program}

1. "Normal Incidence Reflectance: A Robust Tool for In Situ Real-Time Measurement of Growth Rates and Optical Constants of CVD-Grown Semiconductor Thin Films", W. G. Breiland, T. M. Brennan, H. C. Chui, B. E. Hammons, and K. P. Killeen, in "Process Control, Diagnostics, and Modeling in Semiconductor Manufacturing", M. Meyyappan, D. J. Economou, and S. W. Butler, eds, The Electrochemical Society, Inc. Proc. Vol 95-2, 1995, pp 261-269.

2. "A Virtual Interface Method for Extracting Growth Rates and High Temperature Optical Constants from Thin Semiconductor Films using In Situ Normal Incidence Reflectance", W. G. Breiland and K. P. Killeen, J. Appl. Phys., 78,6726 (1995).

3. "Theoretical Study of the Thermochemistry of Molecules in the Si-O-H System", M. D. Allendorf, C. F. Melius, P. Ho, and M.R. Zachariah, J. Physical Chemistry, 99, 15285 (1995).

4. "GCMS and FTIR Studies of By-Product Inhibited Growth and the Rate-Limiting Step in TEOS-Based $\mathrm{SiO}_{2} \mathrm{CVD}$, M.E. Bartram and H.K. Moffat, Proceedings of the Electrochemical Society's Spring Symposium, May, 1995 in Reno, $\mathrm{NV}$, (in press).

5. "Gas Chromatography-Mass Spectroscopy Study of tert-Butylarsine Stability and Purification", M. E. Bartram, W. G. Breiland, K. P. Killeen, and L. A. Bruskas, J. Crystal Growth, (submitted).

6. "TEOS Reaction Rates on $\mathrm{SiO}_{2}$ at 1000K: Zero-Order Dependence on Hydroxyl Coverage and Implications for Reactions With Three-Membered Siloxane Rings", M.E. Bartram and H.K. Moffat, Proceedings of the National AVS Symposium, October, 1995 in Minneapolis, MN, Journal of Vacuum Science and Technology (submitted).

\section{Work performed jointly with other programs, including DP/TTI supported CRADAs}

7. "High Purity GaAs and AlGaAs Grown Using Tertiarybutylarsine, Trimethylaluminum, and Trimethylgallium", R. M. Biefeld, H. C. Chui, B. E. Hammons, W. G. Breiland, T. M. Brennan, E. D. Jones, M. H. Kim, P. Grodzinski, K. H. Chang, and H. C. Lee, J. Crystal Growth, (accepted).

8. "Tertiarybutylarsine for Metalorganic Chemical Vapor Deposition Growth of High Purity Device Quality Materials", H. C. Chui, R. M. Biefeld, B. E. Hammons, W. G. Breiland, T. M. Brennan, and E. D. Jones, J. Electron. Mat. (submitted).

9. " $\mathrm{CBr}_{4}$ for Metalorganic Chemical Vapor Deposition Growth of Carbon-Doped Vertical Cavity Surface-Emitting Lasers, H. C. Chui, B. E. Hammons, K. D. Choquette, and W. G. Breiland, Appl. Phys. Lett (submitted).

10. "The Adsorption and Decompositon of Tertiarybutylarsine on GaAs(100)" C. M. Truong, J.Creighton, submitted to Surface Sci:

11. "A Simplified Analytical Model of Diamond Growth in Direct Current Arcjet Reactors," D. S. Dandy and M. E. Coltrin, J. Mater. Rs., 10, 1993 (1995).

12. "Models of Transport, Gas-Phase and Surface Chemistry in Diamond Chemical Vapor Deposition," D. S. Dandy and M. E. Coltrin, in Proceedings of the Diamond Materials Symposium, edited by K. V. Ravi (Electrochemical Society, Pennington, NJ, 1995, p. 38.)

13. "Monitoring of MOCVD Reactants by UV Absorption, K. C. Baucom, K. P. Killeen and Moffat, submitted to J. Elec. Mat., May, 1995.

14. "Group V Inhibition of GaAs and AlAs MOCVD Growth Rates, H. K. Moffat, K. P. Killeen and K. C. Baucom, submitted to J. Crystal Growth, June, 1995.

15. "The Role of HCl Added to a Dichlorosilane/Ammonia LPCVD Process", P. Ho and W. G.Houf, technical report for CRADA 1082, SAND95-1321, 7/1995. 


\section{Synthesis and Processing of Nanoclusters for Energy Applications \\ KC 020303}

Principal Investigators: J.P. Wilcoxon, J.E. Martin, D. Bliss, P.P. Newcomer, E. Venturini, G.A. Samara and T.R. Thurston (BNL).

Goals: Develop synthesis and processing methods for producing chemically pure, highly crystalline metal and semiconductor nanoclusters with controlled sizes. Investigate the physical properties of these nanoclusters of importance to energy applications such as catalysis.

Recent Highlights:

- Considerable emphasis was placed on improving the synthesis and purification of metal sulfide nanoclusters as potential low-cost, disposable photocatalysts for solar fuel production and detoxification. High quality, monodisperse, crystalline nanoclusters of $\mathrm{MoS}_{2}, \mathrm{FeS}_{2}$ and $\mathrm{PbS}$ were grown in inverse micelles and some of their structural and optical properties investigated. Some of this work was performed jointly with LDRD-funded research.

- The photophysics of electron transfer dynamics of quantum confined $\mathrm{MoS}_{2}$ nanoclusters have been investigated by static and time-resolved emission spectroscopy (w/Kelley-CSU). We have shown that the rates of electron transfer to normal and substituted bipyridine molecules can be controlled through the cluster size. Essentially $100 \%$ reduction of bipyridine (requiring a potential of $\sim 1.5$ volts) to form radical bipyridine anion occurs at room temperature under fluorescence illumination.

- Results on PbS nanoclusters capped with gold have shown that surface related states of one material can be excited through states of a different interior material. This effect has extremely important ramifications for photocatalysts since the functions of energy absorption and energy transfer can be separated. Materials for the interior of the QD can be chosen to be efficient absorbers of light energy, while materials on the surface can be chosen to be efficient at transferring energy to reactants.

- Remarkable structure in the absorption spectrum of $\mathrm{FeS}_{2}$ nanoclusters is observed and large blue shifts in the absorption edge and other spectral features are observed upon crossing from the weak to strong quantum confinement regimes with decreasing cluster size. The bandgap appears to remain indirect down to the smallest cluster size studied ( $2 \mathrm{~nm})$.

- We have observed, for the first time, photoluminescence from metal nanoclusters. This remarkable phenomenon occurs only when the size of the clusters is sufficiently small $(\leq 5 \mathrm{~nm})$ and depends on the nature of the cluster surface and passivating agent. Large, non-luminescent gold clusters can be made photoluminescent by dissolution to smaller sizes.

- In an effort to develop methods to link and assemble nanoclusters, a metal "gel" consisting of metal nanoclusters linked with bifunctional thiols has been formed. TEM shows that the metal clusters are linked but not aggregated, thus retaining their high surface area. Potential applications include a new type of catalytic material capable of fixed bed attachment and recovery.

- $\mathrm{Fe}, \mathrm{Ni}$ and $\mathrm{Co}$ nanoclusters have been synthesized and purified by chromatography. Current efforts are aimed at controlling their oxidation.

Future Directions: Emphasis will be on studying the optical, magnetic and catalytic properties of purified nanoclusters to understand their inherent behavior and the influence of passivating agents and embedding media on these properties. Other specific planned activities include: extending the electron and hole transfer and kinetics studies to $\mathrm{MoSe}_{2}, \mathrm{WS}_{2}$ and $\mathrm{WSe}_{2}$ as these layered semiconductor clusters are among the most viable photocatalysts because of their resistance to photocorrosion. Setting up a reactor with GC/MS for catalytic testing. Studies of the hydrogenation of linear alkene, cyclic alkene, and aromatic molecules using $\mathrm{Pd}$ and $\mathrm{Rh}$ clusters purified by chromatography and comparison with standard commercial catalysts. Attachment of the metal nanoclusters to standard substrates such as $\mathrm{SiO}_{2}$ and $\mathrm{Al}_{2} \mathrm{O}_{3}$ for use as supported catalysts.

Interactions: Defense Programs supports complementary efforts related to potential device applications and on potential use of inverse micelles for environmental remediation. Collaborative Research with Colorado State University and with BNL on hydrogen storage in nanoclusters. Program participates in S\&P Center Project on Nanoscale Materials for Energy Applications.

Program Quality: 5 publications, 2 presentations, Invited presentation and paper to the 1995 International Symposium on Advanced Luminescent Materials. 


\section{Synthesis and Processing of Nanoclusters for Energy Applications \\ KC 020303}

\section{Publications}

Performed Jointly with LDRD funding.

1. F. Parsapour, D.F. Kelley, S. Craft, and J.P. Wilcoxon, "Electron Transfer Dynamics in $\mathrm{MoS}_{2}$ Nanoclusters: Normal and Inverted Behavior," J. Chem. Phys., in press (1996).

2. J.P. Wilcoxon, G.A. Samara, D.E. Bliss and P.P. Newcomer, "Optical Properties of Semiconductor/-Metal Nanoclusters Synthesized in Inverse Micelles," proceedings of the International Symposium on Advanced Luminescent Materials, Chicago, IL (1995).

3. J.P. Wilcoxon, G.A. Samara, and P. Newcomer, "Strong Quantum Confinement effects in Semiconductors: FeS 2 Nanoclusters," Solid State Communications, in press (1996).

4. J.P. Wilcoxon, S. Craft and T. Thurston, "Performance of a High Resolution Bonse-Hart SAXS Instrument using a Synchrotron Source," Rev. Sci. Instruments, (1996).

5. D.E. Bliss, J.P. Wilcoxon, P.P. Newcomer, and G.A. Samara, "Extreme Quantum Confinement and Molecular Features in PbS Nanoclusters," J. Phys. Chem. (submitted). 


\section{Field-Structured Anisotropic Composites \\ KC 020105}

Principal Investigators: J. E. Martin, R. A. Anderson, and C. P. Tigges,

Goals:

We will develop the modeling, synthesis, and processing capability to create novel anisotropic polymer/ceramic and polymer/metal composite materials by applying external electric or magnetic fields to systems consisting of a polymerizable continuous phase into which particles having an electric permittivity or magnetic permeability mismatch are suspended.

\section{Recent Highlights:}

Although this is a new initiative, we have obtained some seed money from the LDRD program and have produced the following results.

- We have developed a molecular dynamics algorithm that simulates the evolution of structure in dielectric or magnetic spheres exposed to a linear or rotating electric field. This algorithm is fully linear in the number of spheres $\mathrm{N}$ and can easily run 10,000 spheres out to $50 \mathrm{~ms}$ in a couple of days. The algorithm is based on induced dipolar interactions and Stokes drag.

- We have developed algorithms that allow us to compute the dielectric constant, structural anisotropy, and scattering functions of the field-structured composites we have simulated. We have used these algorithms to determine the evolution of anisotropy in composites over a wide range of concentration of the dispersed phase.

- We have sythesized a variety of field-structured materials, using an epoxy as the polymerizable continuous phase. Dispersed phases include dielectrics such as barium titanate, strontium titanate, and titania; and magnetic particles of magnetite.

- We have studied the dielectric anisotropy of the materials we have synthesized and the dependence of this on concentration of the dispersed phase, over a volume fraction range from 0.02 to 0.40 . The maximum anisotropy occurs at a volume fraction of 0.20 , and this agrees well with our simulations.

- We have studied the magnetic anisotropy of our materials and have found that the remnant magnetization is very high along the field axis. This may be due to domain alignment within the particles, or to many body effects.

\section{Future Directions:}

Improvements to the molecular dynamics code will include multipolar interactions, lubrication forces, and Brownian motion. We will synthesize samples in rotating fields, as we have discovered that this inverts the anisotropy in the materials, relative to cure in a uniaxial field. Optical and electron microscopy will be used to characterize structure qualitatively when possible. Quantitative, ensemble averaged results will be obtained from high resolution X-ray scattering on our the Bonse-Hart camera at the National Light Source at Brookhaven. We will explore the effect of the quench path on the characteristic domain size in the sample, and will use light scattering to probe structural evolution in concentrated suspensions in an electric field. 


\section{Distribution}

\section{External:}

20 Dr. Iran L. Thomas, Director

Division of Materials Sciences

Office of Basic Energy Sciences

ER-13

U.S. Department of Energy

19901 Germantown Road

Germantown, MD 20874

\section{Internal:}

\begin{tabular}{|c|c|c|c|c|}
\hline MS-0149 & D. L. Hartley, 4000 & 1 & MS-1421 & E. L. Venturini, 1153 \\
\hline MS-0159 & J. S. Binkley, 4531 & 1 & MS-1423 & G. C. Osbourn, 1155 \\
\hline MS-0333 & A. Hurd, 1841 & 1 & MS-1423 & G. N. Hays, 1128 \\
\hline MS-0513 & R. J. Eagan, 1000 & 1 & MS-1427 & P. L. Mattern, 1100 \\
\hline MS-0601 & P. Esherick, 1126 & 1 & MS-1434 & G. E. Pike, 1802 \\
\hline MS-0603 & J. Y. Tsao, 1311 & 1 & MS-1435 & H. J. Saxton, 1800 \\
\hline MS-1056 & B. L. Doyle, 1111 & 1 & MS-9056 & G. A. Fisk, 8355 \\
\hline MS-1079 & A. D. Romig, 1300 & 1 & MS-9161 & W. G. Wolfer, 8717 \\
\hline MS-1349 & R. E. Loehman, 1808 & 1 & MS-9163 & W. Bauer, 8302 \\
\hline MS-1349 & W. F. Hammetter, 1846 & 1 & MS-9405 & M. T. Dyer, 8700 \\
\hline MS-1405 & P. F. Green, 1845 & 8 & MS-1421 & G. A. Samara, 1152 \\
\hline MS-1412 & E. Chason, 1112 & & & \\
\hline MS-1413 & G. Kellogg, 1114 & & & \\
\hline MS-1413 & T. A. Michalske, 1114 & 1 & MS-9018 & Central Tech Files, 8523-2 \\
\hline MS-1415 & P. Gourley, 1112 & 5 & MS-0899 & Technical Library, 4414 \\
\hline MS-1415 & S. M. Myers, 1112 & 1 & MS-0619 & Print Media, 12615 \\
\hline MS-1415 & S. T. Picraux, 1112 & 2 & MS-0100 & Document Processing, 7613-2 \\
\hline MS-1421 & D. Emin, 1153 & 44 & & DOE/OSTI: UC-404 \\
\hline
\end{tabular}

